Submitted to ApJ, 6 DeC 2007

Preprint typeset using LTEX style emulateapj v. 08/22/09

\title{
THERMAL BALANCE IN THE INTRACLUSTER MEDIUM: IS AGN FEEDBACK NECESSARY?
}

\author{
Charlie Conroy \& Jeremiah P. OSTRIKER \\ Department of Astrophysical Sciences, Princeton University, Princeton, NJ 08544 \\ Submitted to ApJ, 6 Dec 2007
}

\begin{abstract}
A variety of physical heating mechanisms are combined with radiative cooling to explore, via one dimensional hydrodynamic simulations, the expected thermal properties of the intracluster medium (ICM) in the context of the cooling flow problem. Energy injection from type Ia supernovae, thermal conduction, and dynamical friction (DF) from orbiting satellite galaxies are considered as potential heating mechanisms of the central regions of the ICM, both separately and in conjunction. The novel feature of this work is the exploration of a wide range of efficiencies of each heating process. While DF and conduction can provide a substantial amount of energy, neither mechanism operating alone can produce nor maintain an ICM in thermal balance over cosmological timescales, in stark contrast with observations. For simulated clusters with initially isothermal temperature profiles, both mechanisms acting in combination result in long-term thermal balance for a range of ICM temperatures and for central electron densities less than $n_{e} \sim 0.02 \mathrm{~cm}^{-3}$; at greater densities catastrophic cooling invariably occurs. Furthermore, these heating mechanisms can neither produce nor maintain clusters with a declining temperature profile in the central regions, implying that the observed "cooling-core" clusters, which have such declining temperature profiles, cannot be maintained with these mechanisms alone. Supernovae heating also fails to maintain clusters in thermal balance for cosmological timescales since such heating is largely unresponsive to the properties of the ICM. Thus, while there appears to be an abundant supply of energy capable of heating the ICM in clusters, it is extremely difficult for the energy deposition to occur in such a way that the ICM remains in thermal balance over cosmological time-scales. For intracluster media that are not in thermal balance, the addition of a small amount of relativistic pressure (provided by e.g. cosmic rays) forestalls neither catastrophic heating nor cooling. These conclusions are driven largely by the fact that 1) DF heating scales approximately as the gas density, while cooling scales as gas density squared, and thus DF heating cannot generically balance cooling without fine-tuning; 2) conduction acts to erase temperature gradients, while most observed clusters in fact show strong gradients in the inner regions. These results strongly suggest that a more dynamic heating process such as feedback from a central black hole is required to generate the properties of observed intracluster media.
\end{abstract}

Subject headings: cooling flows — galaxies: clusters — hydrodynamics — conduction

\section{INTRODUCTION}

Groups and clusters of galaxies are filled with hot plasma in at least approximate pressure equilibrium with the gravitational potential of their dark matter halo. Observations have demonstrated for decades that the cooling time of central regions of this intracluster medium (ICM) in most ( $>$ $70 \%$ ) clusters is shorter than a Hubble time (e.g. Edge et al. 1992; Peres et al. 1998; Sanderson et al. 2006; Vikhlinin et al. 2006b); indeed, it is often as short as $\sim 0.1-1$ Gyr. In the absence of heating, the ICM will thus cool and flow into the central galaxy at the prodigious rate of hundreds of solar masses per year (Cowie \& Binney 1977; Fabian \& Nulsen 1977; Mathews \& Bregman 1978); see Fabian (1994) for a review.

Since this (catastrophically) cooling gas has never been observed (Peterson et al. 2001, 2003; Tamura et al. 2001), it is now generally supposed that there exists one or more heating mechanisms that maintains the ICM in overall thermal balance. The tension created by the fact that the ICM is not observed to be significantly cooling, despite the short cooling times near the cluster center, has become known as the cooling flow problem.

Many possible heating mechanisms have been investigated, including thermal conduction (e.g. Binney \& Cowie 1981; Tucker \& Rosner 1983; Voigt et al.|2002; Fabian et al. 2002; Zakamska \& Narayan 2003; Kim \& Narayan 2003a;
Voigt \& Fabian 2004; Dolag et al. 2004; Pope et al. 2006) which carries heat from the abundant thermal reservoir of the cluster gas to the cooling inner parts, gravitational heating, including the heating due to the orbital motions of galaxies, i.e. dynamical friction (DF; e.g. Miller 1986; Just et al. 1990; Fabian 2003; El-Zant et al. 2004b; Kim et al. 2005; Dekel \& Birnboim 2007), turbulent mixing (e.g. Deiss \& Just 1996; Kim \& Naravan 2003b; Voigt \& Fabian 2004; Dennis \& Chandran 2005), energy injection from active galactic nuclei (AGN) via bubbles, sound waves, etc. (Binney \& Tabor 1995; Nusser et al. 2006; Binney et al. 2007; Ruszkowski et al. 2004a b; Fuiita \& Suzuki 2005; Mathews et al. 2006; Ciotti \& Ostriker 2001; Voit \& Donahue 2005; Ciotti \& Ostriker 2007), and combinations of conduction and AGN (Ruszkowski \& Begelman 2002; Brighenti \& Mathews 2003; Fujita \& Suzuki 2005) or conduction and cosmic-rays (CRs; Guo \& Oh 2007), or AGN and preheating (McCarthy et al. 2007a). One of the most comprehensive studies to date was undertaken by Brighenti \& Mathews (2002) who included many of the heating mechanisms mentioned above and found that no combination generically reproduced observations. A stringent constraint on the potential heating mechanism is that it must not only supply of order the necessary energy but must also supply it in a way that approximately balances cooling (locally) so that the ICM does not either heat up or cool on cosmological time-scales. 
Many of these mechanisms have been idealized, and it is unclear how effective they would be in observed clusters. For example, though difficult to constrain observationally, it has been suggested that the conductivity in clusters may be much smaller than is required to stem the cooling flows (Markevitch et al.2003; Xiang et al.2007). In addition, while DF heating may be attractive, since there are a plethora of satellite galaxies in clusters, it has yet to be identified as important in hydrodynamical simulations (Faltenbacher et al. 2005), although this may be due to insufficient resolution (Naab et al. 2007). Finally, bubbles from activity in central galaxies may be important (e.g. Churazov et al. 2001; Ruszkowski \& Begelman 2002; Brüggen \& Kaiser 2002), but the mechanism by which they transfer their energy to the cluster gas remains obscure.

Even if one or more of these mechanisms could supply the requisite energy (i.e. result in thermal balance), the mechanism must, in addition, not allow the gas to be thermally unstable (Field 1965; Balbus 1986). In what follows we make extensive use of this distinction between thermal balance, where the net cooling of a given parcel of gas is zero, and thermal stability, which pertains to the ability of a gas to remain in thermal balance in the presence of isobaric perturbations. While attention in the literature has focused on investigating the stability of an ICM in thermal balance (e.g. Kim \& Narayan 2003b), herein we address the more fundamental challenge of maintaining a cluster in thermal balance with one or more of the heating mechanisms described above.

The physical mechanism causing thermal instability is easy to understand. Small regions which are slightly over-dense will radiate more than their surroundings (since cooling per unit volume scales as $\rho^{2}$ ) and, isobarically contracting, find a new equilibrium, which is at a higher density and lower temperature. For the temperature domain in question, this leads to further cooling and a thermal runaway results. If a heating process such as conduction or DF heating exists, there will be a stabilizing influence. But if the process scales as $\rho$ (per unit volume) then the equilibrium will not be stable, so high temperature under-dense regions will heat exponentially and lower temperature over-dense regions will still cool exponentially. However, a non-thermal component (such as cosmic rays or tangled magnetic fields) can in principle suppress this instability (Cen 2005; Guo \& Oh 2007, see also the appendix herein) because the non-thermal pressure partially decouples the hydrodynamic balance from the thermodynamics of the cluster gas. In other words, an ICM with a non-thermal component that radiatively cools will lose less total pressure support than an ICM with no non-thermal component. An ICM with a non-thermal component will thus have to contract less to compensate for the lost thermal pressure support.

Observed clusters can be rather cleanly divided into two categories based on the properties of their ICM. "Cooling core" (CC) clusters are those which have steep temperature drops in their central regions (Sanderson et al. 2006) and metallicity gradients (De Grandi \& Molendi 2001); many $\mathrm{CC}$ clusters have identified radio emission at their centers (Best et al. 2005) and show signs of AGN activity, including observed sound waves (e.g. Fabian et al. 2006) apparently emanating from their center, and bubbles at an average distance of $\sim 20 \mathrm{kpc}$ from the cluster center (Bîrzan et al. 2004). In contrast, non-cooling core (NCC) clusters are approximately isothermal (Sanderson et al. 2006) within $\sim 100 \mathrm{kpc}$ and show little metallicity gradient (De Grandi \& Molendi
2001). CC clusters generally have central cooling times of $0.1-1.0$ Gyr while the cooling times in NCC clusters are generally somewhat higher at $\sim 1$ Gyr (Sanderson et al. 2006). The present work investigates the expected thermal properties of both CC and NCC clusters.

The aim of the present study is not to find one or more heating mechanisms that can offset radiative cooling but rather to understand the general conditions that lead to thermal balance in the ICM for a variety of possible mechanisms. This requires both a heating mechanism (or mechanisms) that can energetically offset radiation, and also maintain long term thermal balance.

The rest of this paper proceeds as follows. In $\$ 2$ the methods are discussed, including the implementation of cooling, supernovae heating, conduction, DF heating, and relativistic pressure, the initial equilibria, and the numerical setup. $\$ 3$ contains the results of a series of numerical experiments where the thermal balance of the ICM is investigated. A discussion and summary of these results can be found in $\$ \$ 4$ and 5. respectively. Throughout we assume $h=0.7$ where $h$ is the Hubble parameter in units of $100 \mathrm{~km} \mathrm{~s}^{-1} \mathrm{Mpc}^{-1}$.

\section{METHODS \& PHYSICAL PROCESSES}

This section reviews the relevant fluid equations, cooling function, and heating mechanisms that will be explored in depth in the following sections. This section also discusses our implementation of a relativistic fluid component, initial equilibria, and the numerical setup.

\subsection{General Equations}

The hydrodynamical equations are:

$$
\begin{gathered}
\frac{d \rho_{g}}{d t}=-\rho_{g} \nabla \cdot \mathbf{v}, \\
\frac{d \mathbf{v}}{d t}=-\frac{1}{\rho_{g}} \nabla P_{t o t}+\mathbf{g}, \\
\frac{d e_{g}}{d t}=-\left(e_{g}+P_{g}\right) \nabla \cdot \mathbf{v}+\Gamma-\Lambda,
\end{gathered}
$$

where $\rho_{g}, e_{g}, P_{g}, P_{\text {tot }}$ and $\mathbf{v}$ are the gas density, internal energy density, gas pressure, total pressure, and velocity, $\mathbf{g}$ is the total gravitational acceleration, and $\Gamma$ and $\Lambda$ are the heating and cooling functions per unit volume. We have explicitly distinguished between the total and gas pressures because below we will allow for the addition of a relativistic fluid that can provide additional pressure support. The equation of state for the gas is:

$$
e_{g}=\frac{1}{\gamma_{g}-1} P_{g},
$$

where $\gamma_{g}=5 / 3$ is the ratio of specific heats.

The gravitational acceleration is the combination of a (passive) dark matter halo, a central $\mathrm{cD}$ galaxy, and the selfgravity of the gas:

$$
g(r)=g(r)_{\mathrm{DM}}+g(r)_{\mathrm{cD}}-\frac{G M_{\mathrm{gas}}(<r)}{r^{2}},
$$

where $g_{\mathrm{DM}}$ is

$$
g(r)_{\mathrm{DM}}=-\frac{2 G M_{0}}{r_{s}^{2}}\left[\frac{\ln (1+\mathrm{x})}{x^{2}}-\frac{1}{x(1+x)}\right],
$$


and is derived from the NFW (Navarro et al. 1997) density profile:

$$
\rho_{\mathrm{DM}}=\frac{M_{0} / 2 \pi}{r\left(r+r_{s}\right)^{2}},
$$

where $G$ is Newton's constant, $x \equiv r / r_{s}, M_{0}$ is the normalization and $r_{s}$ is the scale radius, i.e. the radius at which the density profile scales as $r^{-2}$. For reference, the mass within $2 r_{s}$ is equal to $0.86 M_{0}$ for the above density profile. We fix $r_{s}=460 \mathrm{kpc}$ for comparison to Kim et al. (2005). This value for $r_{s}$ is bracketed by the observational range (Vikhlinin et al. $2006 \mathrm{~b}) . M_{0}$ is allowed to vary as discussed in $\$ 2.4$

The central $\mathrm{cD}$ galaxy mass density profile is taken to be a King profile:

$$
\rho_{\mathrm{cD}}(r)=\frac{\rho_{\mathrm{cD}, 0}}{\left[1+\left(r / r_{\mathrm{cD}}\right)^{2}\right]^{3 / 2}}
$$

where

$$
\rho_{\mathrm{cD}, 0}=\frac{9 \sigma_{\mathrm{cD}}^{2}}{4 \pi G r_{\mathrm{cD}}^{2}} .
$$

In the above equations, $\sigma_{\mathrm{cD}}$ is the central $1 \mathrm{D}$ velocity dispersion of the central galaxy and $r_{\mathrm{cD}}$ is the core radius for the King profile; these parameters are taken to be $200 \mathrm{~km} \mathrm{~s}^{-1}$ and $2.83 \mathrm{kpc}$, respectively, from a fit to the cD galaxy NGC 6166 (Kelson et al. 2002) which is representative of cD stellar density profiles. Note that the King profile is for all practical purposes quite similar to the more conventional de Vaucouleurs profile, but is more analytically tractable. The gravitational acceleration associated with this density distribution is

$$
g_{\mathrm{cD}}(r)=-\frac{9 \sigma_{\mathrm{cD}}^{2}}{r_{\mathrm{cD}}}\left(\frac{1+r^{\prime}\left(1+r^{\prime 2}\right)^{-0.5}}{r^{\prime}\left(r^{\prime}+\sqrt{1+r^{\prime 2}}\right)}-\frac{\ln \left(r^{\prime}+\sqrt{1+r^{\prime 2}}\right)}{r^{\prime 2}}\right),
$$

where $r^{\prime} \equiv r / r_{\mathrm{cD}}$.

Mass that flows through the inner boundary $(1 \mathrm{kpc}$; see below), and is hence no longer followed directly in the simulation, is added to the $\mathrm{cD}$ galaxy. Our results are unchanged if the mass is added to a central black hole instead.

The gas is assumed to be ideal:

$$
P=\frac{\rho_{g} k_{B} T}{\mu m_{\mu}}=\frac{\mu_{e}}{\mu} n_{e} k_{B} T,
$$

where $\mu$ is the mean molecular weight, $m_{\mu}$ is the atomic mass unit, $k_{B}$ is Boltzmann's constant, $n_{e}$ is the electron number density and $T$ is the gas temperature. Throughout, the gas metallicity is taken to be $(1 / 3) Z_{\odot}$ (except in $\$ 2.4$ where we discuss the sensitivity of our results to different metallicities).

\subsection{Heating \& Cooling}

This section describes the various heating and cooling mechanisms that are potentially relevant for the thermodynamics of the ICM. Each mechanism includes an adjustable free parameter that is meant to encapsulate both our ignorance regarding the applicability of the mechanism to real clusters and the uncertain values of the particular parameters relevant for each mechanism; a summary of these parameters is provided in Table 1

\subsubsection{Radiation}

A simplified cooling function is adopted that broadly captures the metallicity dependent features in the detailed cooling function of Sutherland \& Dopita (1993) via the following

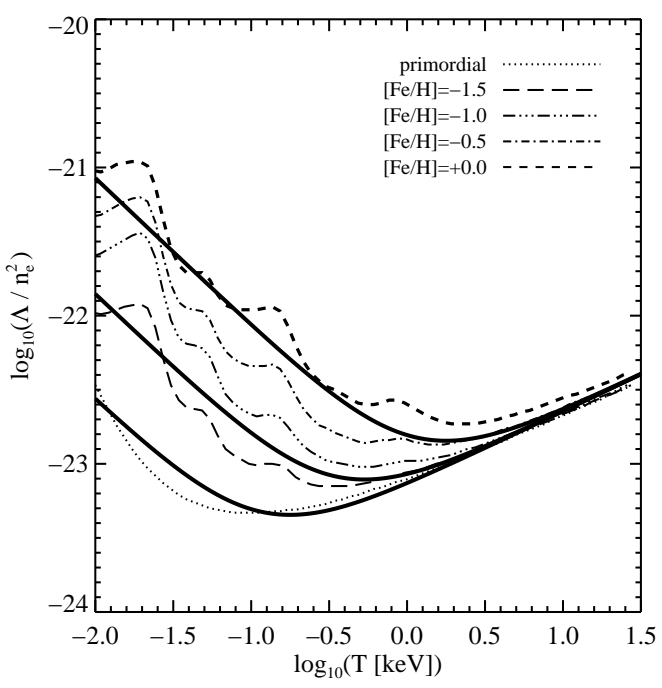

FIG. 1.- Cooling function used in the simulations (solid lines) compared to the more accurate cooling functions of Sutherland \& Dopita (1993). The solid lines are, from bottom to top, for primordial metallicity, $[\mathrm{Fe} / \mathrm{H}]=-1.5$ and $[\mathrm{Fe} / \mathrm{H}]=0.0$. Note that even for low metallicity the cooling function deviates from pure free-free cooling (where $\Lambda \propto T^{1 / 2}$ ) at $T \gtrsim 10^{7} \mathrm{~K}$ due to recombination cooling.

function:

$$
\Lambda=2.1 \times 10^{-27} n_{e}^{2} T^{1 / 2}\left[1+\left(\frac{1.3 \times 10^{7} \zeta(Z)}{T}\right)^{1.5}\right],
$$

in units of $\mathrm{erg} \mathrm{s}^{-1} \mathrm{~cm}^{-3}$. The variable $\zeta$ is a simple function of the gas metallicity, $Z$. This metallicity-dependent cooling function is shown in Figure 1 (solid lines) along with the detailed cooling functions from Sutherland \& Dopita (1993). The cooling function is truncated at $T=0.01 \mathrm{keV}$ because our analytic approximation becomes inaccurate at lower temperature, and because more complex physical phenomena not included herein (such as star formation) become relevant at such temperatures. Most of our results focus on a single metallicity of $Z=\frac{1}{3} Z_{\odot}$; in 2.4 we briefly discuss how the results change when different metallicities are adopted.

\subsubsection{Supernovae}

The stellar populations of central galaxies are extremely old, with typical formation epochs at $z>3$ (Thomas et al. 2005); there are thus no type II supernovae events in these old systems. However, type Ia supernovae events are common, even for the old stellar populations comprising $\mathrm{cD}$ galaxies. At low redshift, roughly one type Ia supernovae event occurs every 100 years per $10^{12} M_{\odot}$ of old stars (Mannucci et al. 2005). Since each supernova releases $\sim 10^{51} \mathrm{ergs}$, which we assume is transfered entirely to the ICM, this corresponds to a

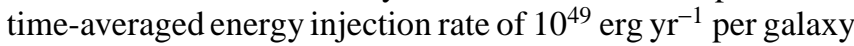
of mass $10^{12} M_{\odot}$. The type Ia rate for a co-evolving stellar population may be a decreasing function of time, indicating that the rate at earlier epochs would be higher than the value we adopt here (Greggio 2005; Mannucci et al. 2006). For our purposes we are primarily interested in type Ia rates at $>1$ Gyr after the formation epoch of the stars, precisely where the time-dependence of the rates are least certain (Mannucci et al. 
2006). However, even a modest increase of the rate with redshift would not qualitatively change our conclusions. We therefore do not include any time-dependence in the type Ia rate in what follows.

Since there are hundreds of galaxies within clusters, supernovae-related energy injection should be treated throughout the cluster. However, since radiation is capable of substantially cooling only the inner cluster region (because cooling scales as $\rho^{2}$ at fixed temperature), and since the thermal reservoir in the outer regions is large, we only distribute supernovae within the central galaxy for simplicity. The supernovae energy injection rate is distributed with the same space density as the $\mathrm{cD}$ galaxy (cf. Equation 8).

Supernovae energy injection is non-negligible in the central regions at the beginning of our simulations. For a initially isothermal cluster with central electron density $0.020 \mathrm{~cm}^{-3}$ and $T_{i}=6 \mathrm{keV}$, the volume-averaged supernovae heating initially dominates cooling within only $2 \mathrm{kpc}$, while for a central density of $0.005 \mathrm{~cm}^{-3}$ supernovae heating dominates within $\sim 10 \mathrm{kpc}$. However, since the injection rate is independent of the properties of the ICM, it has little impact on the long-term thermal balance of the ICM. In particular, while for very low density intracluster media supernovae heating is comparable to radiative cooling on small scales, it is completely ineffective at larger scales (i.e. several tens of $\mathrm{kpc}$ ) because there are so few stars, and hence so few supernovae, at these larger scales.

We have run many simulations for a variety of initial conditions and additional heating sources (discussed below) with supernovae feedback and indeed find that while at the central regions $(\sim 1-10 \mathrm{kpc})$ it can be important, it has little effect on the long-term thermal balance of the ICM. These conclusions are qualitatively similar to those of Kravtsov \& Yepes (2000) who used the observed metallicity of the ICM to demonstrate that supernovae heating is insufficient to offset radiative losses. In order to simplify the discussion in the following sections, we neglect this energy injection process for the remainder of this work.

\subsubsection{Conduction}

The heat flux due to electron conduction may be described by:

$$
\Gamma_{\text {cond }}=\nabla \cdot(\kappa \nabla T),
$$

where $\kappa$ is the conductivity. For a fully ionized plasma, the conductivity is:

$$
\kappa=f \kappa_{S p}=f \frac{1.84 \times 10^{-5} T^{5 / 2}}{\ln \Lambda_{\mathrm{C}}} \operatorname{erg~s}^{-1} \mathrm{~K}^{-1} \mathrm{~cm}^{-1},
$$

where $\kappa_{S p}$ is the classical Spitzer (1962) conductivity, $\ln \Lambda_{\mathrm{C}} \sim$ 37 is the Coulomb logarithm, and $f$ is a free parameter describing the suppression of conductivity relative to the full Spitzer value. While it is difficult to constrain the globally averaged value of $f$ in observed clusters, the strength of observed temperature gradients in several clusters suggests that $f \ll 1$ (Markevitch et al. 2003; Xiang et al. 2007).

There are many theoretical reasons to expect that $f<1$. The primary uncertainty on the importance of conduction is the strength and order of magnetic fields in the ICM. Magnetic fields act to suppress conduction across magnetic field lines (due to the small gyro-radius of electrons) while permitting conduction along the field lines. Observations suggest that the magnetic fields in clusters are tangled with length scales $\sim 1-10 \mathrm{kpc}$ (e.g. Taylor et al. 2002). Tangled magnetic fields or other magnetic phenomena tend to suppress the thermal conductivity to a value roughly $10-30 \%$ of the fully Spitzer value (e.g. Malyshkin \& Kulsrud 2001; Narayan \& Medvedev 2001; Chandran \& Maron 2004), although turbulence may boost the effective conductivity (Cho \& Lazarian 2004). The amount of suppression depends in detail on the poorly constrained properties of the cluster magnetic field, and should thus be considered rather uncertain. Finally, it should be kept in mind that three-dimensional simulations of conduction in the presence of magnetic fields display new instabilities (e.g. Parrish \& Stone 2007) that cannot be captured in our one-dimensional treatment where the conduction is by necessity isotropic. These considerations lead us to adopt $0.0<f<0.5$ as a plausible range for suppression of isotropic conduction (see Table 1 ).

\subsubsection{DF Heating}

A wealth of energy is stored in the orbital motions of galaxies within clusters. DF provides a way to transfer that energy to the background matter, including both the dark matter and the ICM, and can be a potential mechanism that balances the cooling flow, as first pointed out by Miller (1986). Recently, DF heating has experienced a resurgence in popularity (El-Zant et al. 2004b; Kim et al. 2005; Kim 2007) thanks in part to detailed calculations of the efficiency of DF in a collisional medium (Ostriker 1999), which have been verified by controlled numerical experiments (Sánchez-Salcedo \& Brandenburg 2001; Kim \& Kim 2007). These calculations showed that DF heating is stronger in collisional media (such as the ICM) when galaxies are moving slightly supersonically, as appears to be the case in clusters (Faltenbacher et al. 2005), where the average Mach number of galaxies is $\sim 1.3$. DF will also heat the background dark matter halo, thereby producing a core-like inner density profile, as opposed to the cusp implied by the NFW distribution (El-Zant et al. 2004a), although this appears to depend on the detailed properties of the accreted objects (Boylan-Kolchin \& Ma 2007).

A simple calculation suggesting that DF heating may be important in real clusters will be presented first, followed by a more detailed discussion of our implementation of DF heating.

$X$-ray luminosities of clusters vary from $\sim 10^{43}$ to $\sim 10^{45}$ $\operatorname{ergs~s}{ }^{-1}$, with of order $10 \%$ emitted within the rapidly cooling inner region (Fabian 1994). Assuming an average $X$-ray luminosity of $10^{44} \mathrm{ergs} \mathrm{s}^{-1}$, this implies a loss of energy from $X$-ray radiation over a Hubble time of $\sim 5 \times 10^{60}$ ergs within the cooling inner region (e.g. Fabian 1994). The energy liberated during the build-up of the massive central cD galaxy is a plausible source of energy that may balance these radiative losses. The following is a simple calculation demonstrating that the binding energy of the $\mathrm{CD}$ absorbed by the ICM is of the same order as the energy radiated away within the cooling region.

The energy liberated during the build-up of the $\mathrm{cD}$ galaxy is

$$
\Delta E=\int \phi \mathrm{d} m_{*}=4 \pi \int \phi \rho_{*} r^{2} \mathrm{~d} r,
$$

where $\Delta E$ is the binding energy, $\phi$ is the total gravitational potential felt by the $\mathrm{cD}, m_{*}$ is a unit of stellar mass, and $\rho_{*}$ is 
the stellar density profile. We assume that $\phi$ is static for simplicity, though of course the dark matter halo is being built-up at the same time as the $\mathrm{cD}$.

The $\mathrm{cD}$ galaxy projected density profile is approximated as a Sersic profile (Sersic 1968), in agreement with a variety of observations. The projected Sersic profile is not analytically invertible, but sufficiently accurate fitting functions exist in the literature. The de-projected stellar mass density can be approximated by (Lima Neto et al. 1999):

$$
\rho_{*}(r)=A s^{-\alpha} \exp \left(-b_{n} s^{1 / n}\right)
$$

where $s \equiv r / R_{e}, b_{n} \approx 2 n-0.327$, and $\alpha \approx 1-1.188 /(2 n)+$ $0.22 /\left(4 n^{2}\right) . A$ is an integration constant chosen such that the integral over $\rho_{*}$ equals the total stellar mass $M_{*}$.

For simplicity the total gravitational potential within the inner regions is assumed to be isothermal:

$$
\phi(r)=\mathrm{V}_{c}^{2} \ln \left(r / r_{\text {cool }}\right),
$$

where $\mathrm{V}_{c}$ is the circular velocity. We are only interested in energy liberated within the cooling region $\left(r<r_{\text {cool }}\right)$, and so set the potential to be zero at $r=r_{\text {cool }}$. Define $\gamma \equiv r_{\text {cool }} / R_{e}$.

Combining these results yields:

$$
\Delta E=V_{c}^{2} M_{*} \frac{\int_{s}^{0} \ln \left(\frac{s^{\prime}}{\gamma}\right) s^{\prime 2-\alpha} \exp \left[-b_{n} s^{\prime 1 / n}\right] \mathrm{d} s^{\prime}}{\int_{0}^{s} s^{\prime 2-\alpha} \exp \left[-b_{n} s^{\prime 1 / n}\right] \mathrm{d} s^{\prime}} .
$$

The two integrals represent an average of the logarithmic run of the potential over the stellar density profile. The integrals are functions only of the Sersic index $n$, the ratio between the cooling radius and the effective radius $\gamma$, and the upper limit of integration $s$ (taken to be the same in both integrals, which is reasonable but not necessary). For $1<s<3$ and $4<n<10$ the ratio of these two integrals varies from 0.4 to 1.8 , for $\gamma=1$. The integral scales roughly as $\sqrt{\gamma}$.

For a typical cD galaxy $M_{*} \sim 10^{12} M_{\odot}$ and for a typical cluster $\mathrm{V}_{c} \sim 700 \mathrm{kms}^{-1}$ (Gonzalez et al. 2007) implying that $\Delta E \sim 10^{61}$ ergs. This energy can be transferred into both the background ICM and dark matter halo. If the gas were collisionless, then the fraction of energy shared between the gas and the dark matter would be simply proportional to their relative mass fractions $(\sim 1 / 6$ and $\sim 5 / 6$ for the gas and dark matter respectively). However, the collisional nature of the gas makes DF more efficient at transonic speeds (Ostriker 1999); at Mach numbers near unity the efficiency of DF heating on the gas is roughly twice that of the collisionless dark matter. This implies that $\sim 30 \%$ of the energy released in transferred to the ICM and the rest to the dark matter. The $\mathrm{cD}$ binding energy thus absorbed by the ICM is comparable to the energy radiated away within the cooling inner region over a Hubble time.

There were many simplifications made in this calculation. One complication is that observations indicate that a large fraction of massive $\left(M_{*}>10^{11.5} M_{\odot}\right)$ galaxies were already in place by $z \sim 1$ (e.g. Bundy et al. 2005; Borch et al. 2006; Fontana et al. 2006; Brown et al. 2007), and hence the energy liberated from the build-up of cDs may not provide much energy to the ICM at $z<1$. However, the outer envelope of the $\mathrm{cD}$ may be growing substantially at $z<1$ from the shredding of satellite galaxies (see e.g. Ostriker \& Hausman 1977; Conroy et al. 2007; Purcell et al. 2007, for a discussion), suggesting that energy released from the build-up of the $\mathrm{cD}$ is available at late times. Moreover, the strong small-scale clustering of luminous red galaxies indicates that there are many massive galaxies orbiting near the cluster center, plausibly transferring their orbital energy to the ICM and background halo via DF (Masjedi et al. 2006).

These calculations suggest that DF heating may be an important heating mechanism. While the simulations below address DF heating in more detail, detailed high-resolution three-dimensional simulations are required to fully address the importance of DF heating in the ICM of clusters (see e.g. Kim 2007). Moreover, detailed resolution studies are required to address whether or not current cosmological hydrodynamic simulations are adequately resolving DF (see discussion in Faltenbacher et al. 2005; Naab et al. 2007).

Our implementation of DF heating closely follows that of Kim et al. (2005); the reader is referred to that work for more details. Note that we do not track orbits explicitly nor do we attempt to resolve the actual DF wake. We take a more approximate, parameterized approach to the sphericallyaveraged heating rate due to DF.

The heat flux due to DF can be described as:

$$
\Gamma_{D F}=n_{\mathrm{gal}}\left\langle-\mathbf{F}_{\mathrm{DF}} \cdot \mathbf{v}\right\rangle=d \frac{4 \pi \rho_{g} G^{2} \overline{M_{\mathrm{gal}}^{2}}\langle I / \mathcal{M}\rangle}{c_{s}} n_{\mathrm{gal}}(r),
$$

where $\mathcal{M} \equiv \mathbf{v} / c_{s}$ is the Mach number, $n_{\text {gal }}$ is the number density of galaxies, $\overline{M_{\text {gal }}^{2}}$ is the average squared total mass of the galaxies, and $c_{s}$ is the isothermal sound speed. In what follows we will argue for reasonable values for each of the parameters in Equation 19, and will then incorporate the uncertainties and possible ranges in all of these parameters into the single free parameter $d$. The angular brackets indicate an average over the velocity distribution function.

Note that $M_{\mathrm{gal}}$ includes both the stellar and dark matter of the satellite galaxies, and that since DF is proportional to the average of the galaxy mass squared, more massive galaxies are given greater weight. The quantity $\overline{M_{\text {gal }}^{2}}$ is estimated as follows. We assume that the dark matter-to-stellar mass ratio is constant for satellites, and estimate it by taking the total stellar mass of satellites in clusters to be $1 \%$ of the total cluster mass (Gonzalez et al. 2007) and the total amount of dark matter mass in substructures to be $10 \%$ (Gao et al. 2004), where the substructures are assumed to be the likely locations of satellite galaxies (Conroy et al. 2006). These numbers imply an average dark matter-to-stellar mass ratio of 10 for satellite galaxies. If the total mass is $M_{\mathrm{gal}}$, then $\overline{M_{\mathrm{gal}}^{2}}=\overline{M_{\mathrm{star}}^{2}}+\overline{M_{\mathrm{dm}}^{2}}=$ $101 \overline{M_{\text {star }}^{2}}$. The stellar mass function provides an estimate of $\overline{M_{\text {star }}^{2}}$ and thus of $\overline{M_{\text {gal }}^{2}}$. We adopt the global mass function of Bell et al. (2003) where $\alpha=1.1$ and $\log \left(M^{*}\right)=10.9 M_{\odot}$ are the best-fit Schechter parameters. The Schechter parameters for the luminosity function within clusters does not differ strongly from the global value (Hansen et al. 2007), and so we adopt the global values for this calculation. Integrating the stellar mass function to $M_{\text {star }}=10^{8} M_{\odot}$ leads us to adopt $\overline{M_{\text {gal }}^{2}}=\left(10^{11} M_{\odot}\right)^{2}$ as the fiducial value. The uncertainty on this quantity is explored via the tunable parameter $d$ discussed above.

By definition we have:

$$
\langle I / \mathcal{M}\rangle=\frac{\int I / \mathcal{M} f(\mathbf{v}) d \mathbf{v}}{\int f(\mathbf{v}) d \mathbf{v}},
$$

where $f$ is assumed to be Maxwellian (Faltenbacher et al. 
TABLE 1

SUMMARY OF FREE PARAMETERS

\begin{tabular}{lll}
\hline \hline Parameter & \multicolumn{1}{c}{ Comment } & $\begin{array}{c}\text { Plausible } \\
\text { Range }\end{array}$ \\
\hline & & \\
$Z / Z_{\odot}$ & Metallicity of the gas (Eqn. 12] & $0.1-0.6$ \\
$f$ & Fraction of Spitzer conductivity (Eqn. 14 & $0.0-0.5$ \\
$d$ & Normalization of DF heating (Eqn. 19] & $0.1-10.0$ \\
$\alpha$ & Initial fraction of relativistic pressure (Eqn. 23] & $0.0-0.3$
\end{tabular}

2005):

$$
f(\mathbf{v})=\frac{4 \pi N_{\mathrm{gal}}}{\left(2 \pi \sigma_{r}^{2}\right)^{3 / 2}} \mathbf{v}^{2} e^{-\mathbf{v}^{2} /\left(2 \sigma_{r}^{2}\right)},
$$

where $\sigma_{r}$ is the radial velocity dispersion of galaxies (assumed for simplicity to be independent of radius), and $N_{\text {gal }}$ is the total number of galaxies within the cluster. In the following we take $\sigma_{r}=1000 \mathrm{~km} \mathrm{~s}^{-1}$ and $N_{\text {gal }}=500$. These parameters are fixed throughout; any variation of these physical quantities is incorporated into the $d$ parameter. Note that DF heating depends on the state variables only through $c_{s}, I / \mathcal{M}$, and $\rho_{g}$, and that $\Gamma_{D F} \propto \rho_{g}$ at fixed temperature. $I$ is the efficiency factor for DF in the collisional case (Ostriker 1999):

$$
I \equiv \begin{cases}\frac{1}{2} \ln \left(1-\mathcal{M}^{-2}\right)+\ln \left(\mathrm{vt} / \mathrm{r}_{\min }\right) & \mathcal{M}>1 \\ \frac{1}{2} \ln \left(\frac{1+\mathcal{M}}{1-\mathcal{M}}\right)-\mathcal{M} & \mathcal{M}<1\end{cases}
$$

As is common practice, we set $v t=r_{\max }$, where $r_{\max }$ and $r_{\min }$ are in this problem taken to denote the size of the cluster and the satellite galaxies, respectively. The factor $\ln \left(v t / r_{\min }\right)$ thus plausibly varies from $\sim 4-10$. In what follows we set this factor to 6 and note that our results are insensitive to this particular value. Note that DF heating is more efficient at subsonic speeds than one might naively expect because the relevant efficiency is $\langle I / \mathcal{M}\rangle$, which, though maximal at $\mathcal{M}=1$, decreases by only $50 \%$ at $\mathcal{M} \approx 0.5$ and decreases only weakly at $\mathcal{M}>1$. This implies that the feedback provided by the Mach number (in the sense that colder/hotter systems will have more/less efficient DF heating) is weaker than one might have expected.

The number density of galaxies, $n_{\text {gal }}(r)$, is taken to be a modified King profile with the parameters adopted from Girardi et al. (1998):

$$
n_{\text {gal }}(r)=n_{\text {gal }}(0)\left[1+\left(r / r_{c}\right)^{2}\right]^{-1.2}
$$

where the core radius $r_{c}=50 \mathrm{kpc}$. The central density is set by requiring that $n_{\text {gal }}(r)$ integrate to the total number of galaxies, $N_{\text {gal }}$. Note that $n_{\text {gal }}(r)$ does not include the central cD galaxy. While most $\mathrm{cD}$ galaxies are near the center of the halo as defined by $X$-ray imaging (Lin \& Mohr 2004), the cD may be oscillating about the gravitational center with an amplitude of several kpc (van den Bosch et al. 2005). Such oscillations may provide additional heating in the central regions, but we do not include them herein.

\subsection{Relativistic Pressure}

Relativistic pressure in the form of cosmic rays could be an important dynamical component of the ICM. It has long been known that astrophysical shocks efficiently accelerate cosmic rays (Blandford \& Ostriker 1978). Numerical simulations suggest that cosmic rays accelerated in shocks could account for $\sim 10 \%$ of the total cluster pressure (Miniati et al. 2001; Ryu et al. 2003). Cosmic rays may also be generated in buoyantly rising bubbles generated by AGN activity (e.g. Enßlin 2003). Observations suggest that a relativistic component may constitute several tens of percent of the total energy density of clusters (Pfrommer \& Enßlin 2004; Sanders et al. 2005; Dunn \& Fabian 2006; Sanders \& Fabian 2007; Werner et al. 2007; Nakar et al. 2007). In addition, it has been shown that a relativistic pressure component can suppress thermal instabilities (Cen 2005, see also the appendix herein).

There have been several recent attempts to study the effects of cosmic-rays in the ICM numerically (Pfrommer et al. 2007b; ; Pfrommer 2007; Guo \& Oh 2007). The model of Guo \& Oh (2007), which includes both conduction and cosmic rays injected into the ICM from AGN-induced bubbles, is able to reproduce the temperature and density profiles of observed clusters. Perhaps the most attractive feature of their model is that their results do not require fine tuning of the various adjustable parameters, including the amount of thermal conductivity. It would be interesting to know whether and to what extent their results rely on the relativistic pressure provided by the CRs, or whether it is due primarily to the energetics associated with the bubbles. In the present work we test the former hypothesis.

In the following we take a simplified approach when exploring the effects of a relativistic component. The relativistic pressure $\left(P_{r}\right)$ is assumed to be a fixed fraction of the total pressure initially:

$$
P_{r}=\alpha P_{t o t},
$$

and we further assume that the relativistic component is perfectly dynamically coupled to the gas (i.e. there is no motion or diffusion of one component relative to another), and evolves adiabatically (i.e. $P_{r} \propto \rho_{g}^{\gamma_{r}}$ ). These requirements lead to a fourth fluid equation:

$$
\frac{\partial P_{r}}{\partial t}=-\nabla \cdot\left(\mathbf{v} P_{r}\right)+\left(1-\gamma_{r}\right) P_{r} \nabla \cdot \mathbf{v}
$$

which is simply a statement of energy conservation. In the above equation $\gamma_{r}=4 / 3$ and we have made use of the following equation of state:

$$
e_{r}=\frac{1}{\gamma_{r}-1} P_{r}
$$

where $e_{r}$ is the energy density associated with the relativistic fluid. Note that relativistic pressure also enters into the hydrodynamical equations (Equations $1-3$ ) by contributing to the total pressure.

These simple assumptions are motivated by CR creation and evolution in real clusters. It may be the case that CRs are created predominately in the cluster center via AGN activity, or it may be that the accretion shock at the cluster outskirts are the predominate source of CRs. CRs are capable of diffusing out of the cluster, but CR loss via diffusion may be approximately balanced by the creation of new CRs throughout the cluster. Our simplified treatment is meant to demonstrate the potential importance of CRs generally (insofar as they provide relativistic pressure support); it will be the task of more sophisticated modeling efforts (see e.g. Pfrommer et al. 2007b a; Pfrommer 2007; Guo \& Oh 2007) and, ultimately, observations to refine our knowledge of CR production and evolution. Note that the energetics associated with CR pro- 
duction (whether in e.g. shocks or AGN-related bubbles) is not explored herein.

\subsection{Initial Equilibria}

Every simulated cluster is set up initially in hydrostatic equilibrium. However, in general the clusters are not set up in thermal balance.

Observations indicate that there are two classes of intracluster media, the cooling-core (CC) and non-cooling-core (NCC) clusters (Sanderson et al. 2006). Clusters in the former class show a rapidly declining temperature profile with decreasing radius at radii less than $\sim 100 \mathrm{kpc}$, while the latter are approximately isothermal within the same physical region. Furthermore, CC clusters have factors of $\sim 2-3$ metallicity gradients within $\sim 100 \mathrm{kpc}$ while NCC clusters do not (De Grandi \& Molendi 2001). For our analysis we assume that neither NCC nor CC clusters have an appreciable metallicity gradient, and that the metallicity is $(1 / 3) Z_{\odot}$. We have selected several cluster runs at random and re-simulated them with metallicity varying from primordial to solar composition and find no qualitative change in our results.

Both of these classes are discussed in the sections that follow. NCC clusters as approximated as initially isothermal while the $\mathrm{CC}$ clusters are assumed to have an initial temperature profile that is described by (Vikhlinin et al. 2006b):

$$
T(r) / T_{\mathrm{CC}}=\frac{1.15(x / 0.045)^{2}+0.5}{(x / 0.045)^{2}+1} \frac{1}{1+(x / 0.75)^{2}},
$$

where $x \equiv r / r_{500}$. This temperature profile peaks at $T_{\mathrm{CC}}$ where $r \sim 0.1 r_{500}$ and drops by a factor of $\sim 2-3$ toward the center. Figure 2 (bottom panel) provides an example of a $\mathrm{CC}$ cluster temperature profile.

In each run the cumulative gas fraction at $500 \mathrm{kpc}$ is fixed at $f_{g}=0.1$, in agreement with recent observations (Sanderson et al. 2003; Vikhlinin et al. 2006b). The gas fraction is less than the universal baryon fraction of $f_{b}=0.16$ for several reasons (see e.g. Kravtsov et al. 2005, for a discussion). The conversion of gas into stars results in a lower gas fraction by several tens of percent (Fukugita et al. 1998; Lin et al. 2003). Both adiabatic effects and heating processes further decrease the gas fraction within $\sim 500 \mathrm{kpc}$ (Bode et al. 2007). Fixing the gas fraction implies that simulated clusters with larger central electron densities are embedded in larger dark matter halos, as observed (Vikhlinin et al. 2006b).

With the above simplifications, each simulation is fully specified by the initial central electron density and the temperature profile. These two parameters, along with the assumption of hydrostatic equilibrium, then determines the full density, temperature, and pressure profiles, and the adopted gas mass fraction then determines the mass of the static dark matter halo. The contribution of the $\mathrm{cD}$ galaxy to the gravitational potential is fixed initially and increases in proportion to the amount of mass that flows through the inner boundary condition (see 2.1 . In practice we iteratively solve for $M_{0}$ such that hydrostatic balance is achieved. For the central densities explored below, the NFW normalization $M_{0}$ ranges from $\sim(2-7) \times 10^{14} M_{\odot}$. Allowing $M_{0}$ to vary by fixing $f_{g}$ as opposed to allowing $f_{g}$ to vary by fixing $M_{0}$ has a negligible effect on our conclusions.

Observationally the temperature of clusters varies from $\sim$ $1-10 \mathrm{keV}$, and is strongly correlated with the total cluster mass (e.g. Vikhlinin et al. 2006b). The data also cover a wide range in central electron densities, roughly $10^{-2.5}<n_{e, 0}<$

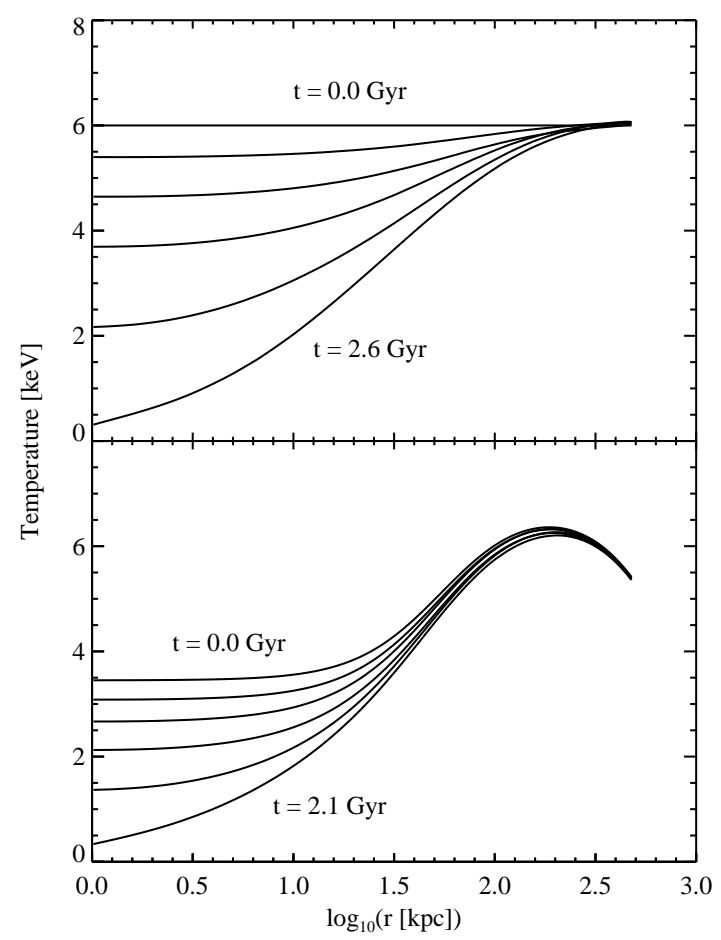

FIG. 2.- Evolution of the temperature profile of clusters with radiative cooling and no heating for $n_{e, 0}=0.02 \mathrm{~cm}^{-3}$. As expected, a runaway cooling flow develops and the core collapses within a cooling time. Top Panel: An initially NCC cluster. Bottom Panel: An initially CC cluster. The lines are spaced equally in time.

$10^{-0.5} \mathrm{~cm}^{-3}$. We pay more attention to how cooling and heating depends on density because the cooling and heating functions are more sensitive to density than temperature. Below we include "observed" central electron densities for comparison with our initial conditions, which are estimated from two sources. Zakamska \& Narayan (2003) provide central densities from hydrostatic fits to observed temperature and density profiles of 10 clusters. Vikhlinin et al. (2006b) provide detailed parametric fits to the electron density profiles of 13 clusters (two clusters are in both samples and are only counted once herein). For the latter sample we quote as the central density the density at $10 \mathrm{kpc}$ because in many cases the density profile at smaller scales is enhanced due to cooling. Since we are interested in "initial" central electron densities, we essentially mask out this inner cooling region when comparing to the initial electron densities in our simulations. In the simulations the initial central electron densities are approximately constant within the inner $10 \mathrm{kpc}$; we thus consider this simplification appropriate.

\subsection{Numerical Setup}

We utilize the Lax-Wendroff method (Press et al. 1992) to integrate the fluid equations in their one-dimensional form. The spatial grid is logarithmic and has $N=400$ elements with range $1<r<500 \mathrm{kpc}$. We have doubled the number of grid elements for several simulations and extended the spatial grid to $1000 \mathrm{kpc}$ and find no change in the results. The time-step is determined by the Courant condition:

$$
\Delta t_{C F L}=0.5 \min \left(\frac{\Delta r_{i}}{\left|\mathbf{v}_{i}+c_{s, i}\right|}\right),
$$

where $i=[0, N]$ and $c_{s, i}$ is the local isothermal sound speed. 


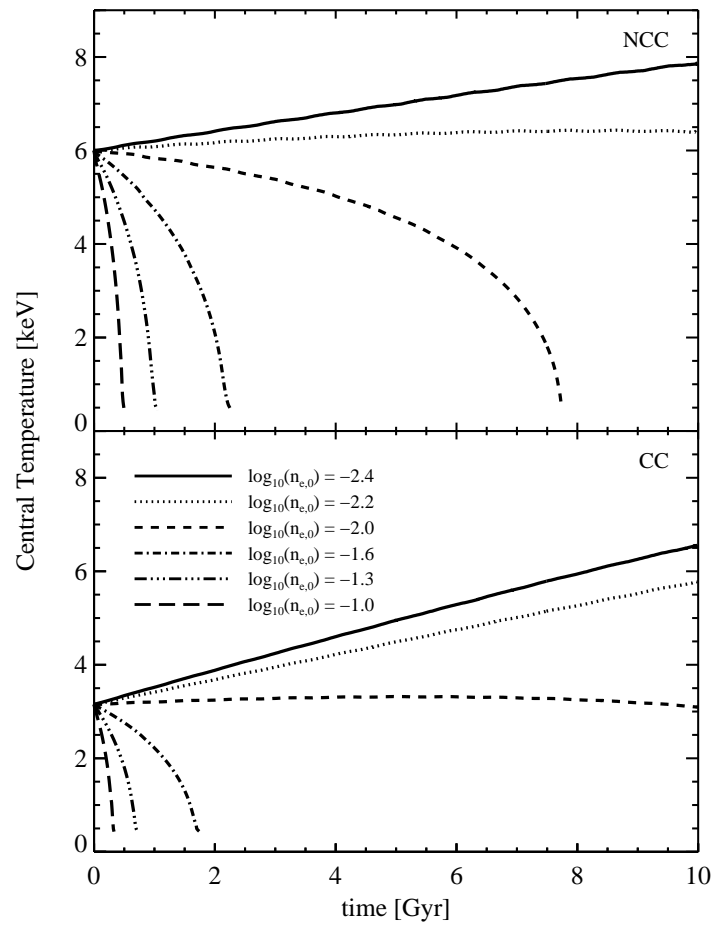

FIG. 3.- Evolution of the central temperature for simulations that include both radiation and DF heating. Each panel shows the evolution of clusters with a variety of initial central densities (labeled in the figure in units of $\mathrm{cm}^{-3}$ ). The normalization of DF heating here is fixed at $d=1.0$ (cf. Equation 19. Top Panel: Initially NCC clusters. Bottom Panel: Initially CC clusters. The evolution of CC clusters is qualitatively similar to NCC clusters in the sense that thermal balance can only be maintained for a narrow range of central electron densities.

Conduction is implemented with a fully implicit algorithm (Press et al. 1992). The pressure, density, and temperature in the ghost cells are linearly extrapolated from the active zones, both at the inner and outer boundaries. At the inner boundary, the velocity is also a linear extrapolation from the active zones unless the velocity in the ghost cell is positive (indicating outflow) in which case the velocity is set to zero. The velocity in the ghost cell at the outer boundary is always zero.

\section{NUMERICAL EXPERIMENTS}

This section presents the results of a series of numerical experiments. The various physical processes discussed in $\$ 2.2$ are discussed in a variety of combinations in order to elucidate their various effects. For each process we discuss the sensitivity of the final state to the free parameters summarized in Table 1.

\subsection{Radiation Only}

As discussed in the Introduction, in the absence of any heating mechanisms the ICM will cool catastrophically. The time required to radiate away all of the internal energy of the gas is defined as the cooling time ${ }^{1}$ :

$$
t_{\text {cool }} \equiv \frac{\rho_{g} e}{\Lambda}=5.0\left(\frac{0.01 \mathrm{~cm}^{-3}}{n_{e}}\right)\left(\frac{T}{6 \mathrm{keV}}\right)^{0.5} \mathrm{Gyr} .
$$

${ }^{1}$ Note that this cooling time differs from the conventional cooling time by a factor of $\frac{\gamma_{g}}{\gamma_{g}-1}$ because the conventional cooling time is defined as the time required to cool isobarically whereas the cooling time in Equation 28] is simply the time required to radiate away all of the thermal energy.
As can be seen from Equation 28, the central cooling time for typical clusters is less than a Hubble time.

Figure 2 shows the evolution of the ICM for two clusters with radiative cooling and no heating. It is clear that the clusters cool catastrophically in the absence of any heating. The top panel shows the evolution of an initially isothermal cluster with $T=6 \mathrm{keV}$ and an initial central electron density $n_{e, 0}=0.02 \mathrm{~cm}^{-3}$; the bottom panel shows the evolution of an initially $\mathrm{CC}$ cluster with the same central electron density. The cooling times for the NCC and CC clusters shown in the figure are 2.4 and $1.7 \mathrm{Gyr}$, respectively, based on Equation 28 and adequately captures the actual time for catastrophic collapse.

While there are clusters with temperature profiles similar to that shown in the top panel of Figure 2] it is important to note that not only are these temperature profiles transient (in the sense that the cluster continues to cool on rapid timescales), but the density profile of this radiation-only cluster (not shown) is nowhere observed in nature. Hence the $X$-ray luminosities, which scale as the gas density squared, would be out of the range of those observed in nature. The conclusion that observed clusters must be periodically heated is in accord with a large body of previous work. In particular, $X$-ray spectroscopy has firmly established that the ICM is not cooling much below $\sim 1 / 3$ of the ambient temperature (Peterson et al. 2001, 2003; ; Tamura et al. 2001).

The following sections discuss possible heating mechanisms that can forestall the cooling catastrophe visible in Figure 2 .

\subsection{Radiation \& DF Heating}

As discussed in 2.2 .4 there is an enormous source of energy in the orbital motions of satellite galaxies. While satellite galaxies can transfer their orbital energy to the ICM through a variety of mechanisms, we will focus only on DF heating for simplicity. Note however, that other mechanisms capable of transferring the orbital energy of satellites to the background gas are expected to scale in a similar way as DF heating (i.e. as $\rho_{g}$ at fixed temperature). The discussion that follows thus roughly encompasses a variety of heating mechanisms related to the motions of satellite galaxies.

Figure 3 shows the evolution of the ICM central temperature, $T_{c}$, as a function of time for initially NCC (i.e. isothermal; top panel) and CC clusters (bottom panel). Each panel shows the central temperature evolution for a range of initial central gas densities. The normalization of DF heating shown in the figure is set to $d=1.0$.

Several trends are apparent. First, it is clear that one can find a particular set of parameters that leads to approximate thermal balance (neither a heating nor cooling catastrophe) over a Hubble time. While not shown, we note that in the runs where the central temperature does not change appreciably, neither do the density nor temperature profiles. This was first demonstrated by Kim et al. (2005). In fact, our fiducial values for DF heating are the same as those in Kim et al. (2005). The equilibrium NCC model has $T_{i, N C C}=6 \mathrm{keV}$ and $n_{e, 0}=6 \times 10^{-3} \mathrm{~cm}^{-3}$ which is exactly the set of equilibrium parameters found in Kim et al. (2005), thereby confirming the results of that work.

It is also apparent that DF heating as the sole heating mechanism leads to serious thermal imbalance for all but a very narrow range of electron densities, as was also demonstrated by Kim et al. (2005). This imbalance arises because at fixed 

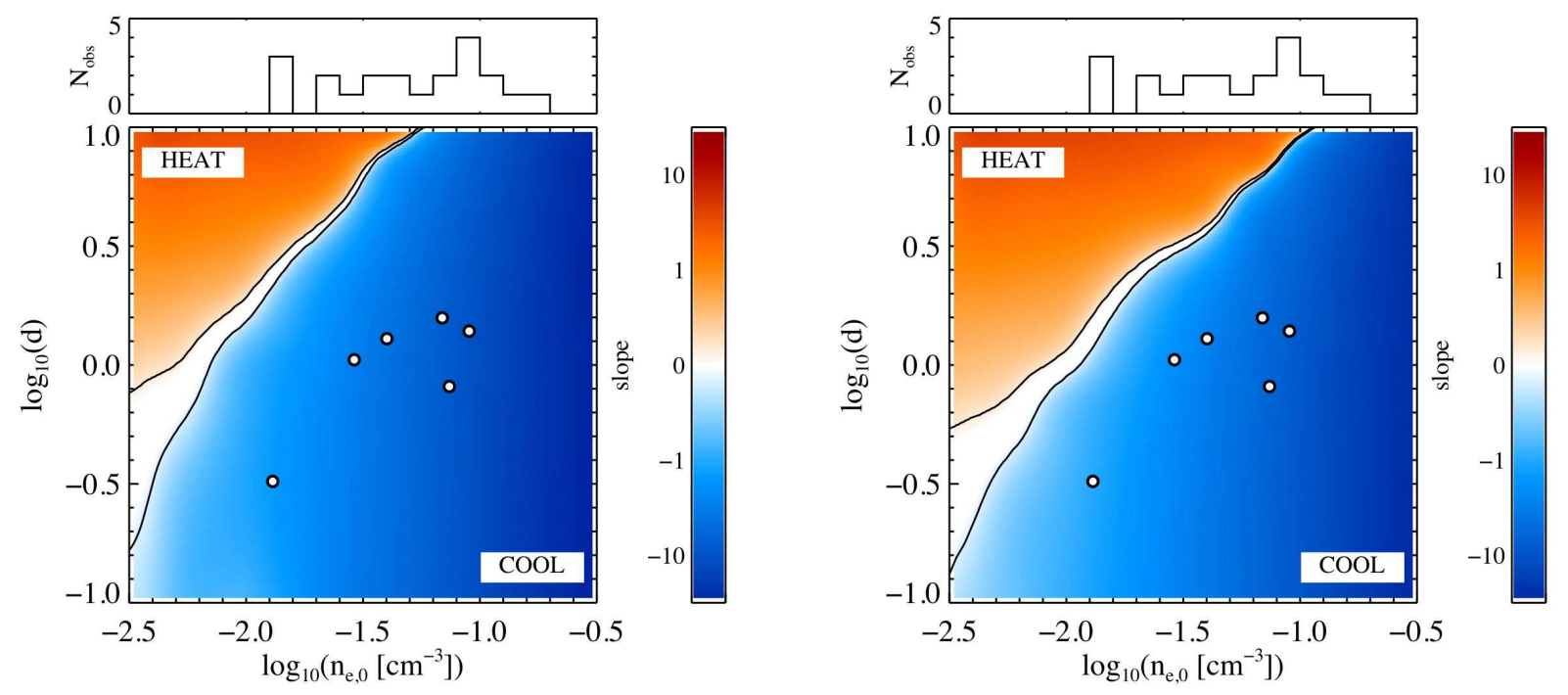

FIG. 4.- Long-term thermal evolution of intracluster media subjected to both DF heating and radiative cooling, as a function of initial central density, $n_{e, 0}$ and normalization of DF heating, $d$ (cf. Equation 19. Both NCC clusters with $T_{i, N C C}=6 \mathrm{keV}$ (left panel) and CC clusters with $T_{i, C C}=6 \mathrm{keV}$ (right panel) are shown. The color indicates the slope of central temperature vs. time. Red indicates the region of parameter space where the cluster gas catastrophically heats and blue where the gas catastrophically cools (i.e. where the slope is positive or negative). The solid black lines indicate the region of parameter space where the slope is equal to \pm 0.1 . Note that while there is a domain where heating stably balances cooling, sensitive fine-tuning is required for a real cluster to remain in this domain. For comparison, the distribution of observed central electron densities is included at the top (see \$2.4) and the estimated location of several Abell clusters are included in the $d-n_{e, 0}$ plane (circles).

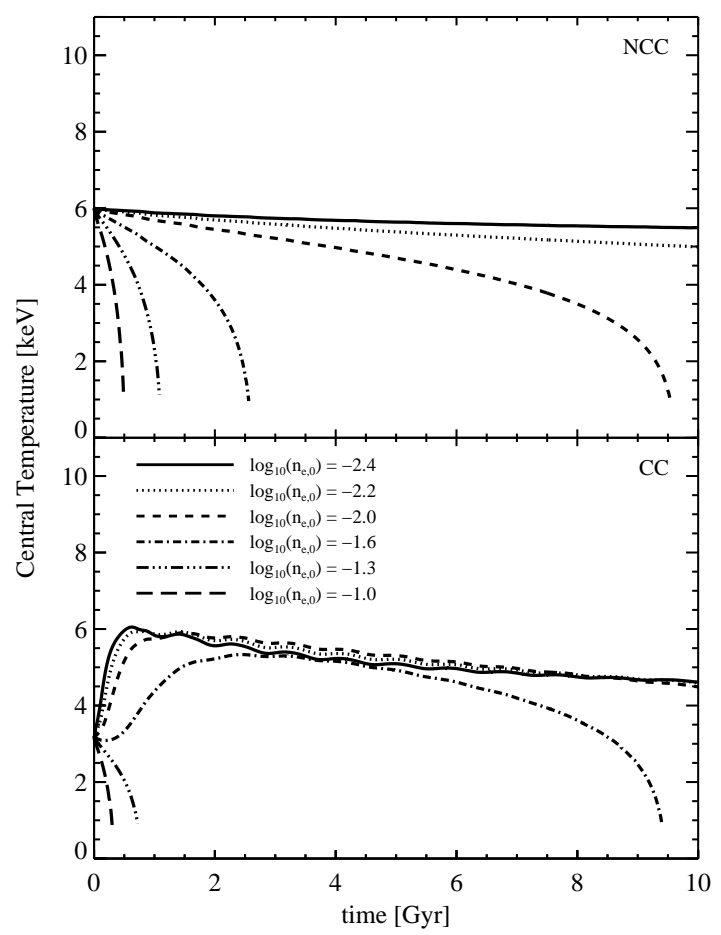

FIG. 5.- Evolution of the central temperature for simulations that include both radiation and conduction. The conduction normalization is $f=0.1$ (cf. Equation 14. Top Panel: Initially NCC clusters with $T_{i, N C C}=6 \mathrm{keV}$. Bottom Panel: CC clusters with $T_{i, C C}=6 \mathrm{keV}$.

temperature the cooling scales as $\rho_{g}^{2}$ while DF heating scales as $\rho_{g}$. Thus at fixed temperature there can be only one initial density that is in thermal balance, and that solution will be unstable.

In order to more fully elucidate the thermal properties of the ICM, Figure 4 shows the long-term thermal evolution of the ICM as a function of initial central density, $n_{e, 0}$ and normalization of DF heating, $d$, for NCC (left panel) and CC (right panel) clusters for a fixed initial temperature profile. In this figure the slope of the central temperature as a function of time is color-coded according to the sign and steepness of the slope. From Figure 3 it is clear that $T_{c}$ for most clusters evolves at least quasi-linearly, and hence the slope is a reasonable metric for the evolution of the ICM.

Figure 4 demonstrates that there is only a narrow range of thermal balance in the parameter space of $d$ and $n_{e, 0}$. This highlights the inability of DF heating operating alone to balance radiative losses in the ICM. The normalization of DF heating that is required to balance radiative losses increases in proportion to the central electron density $\left(d \propto n_{e, 0}\right)$, which makes the DF heating term (Equation 19) effectively scale as $\rho_{g}^{2}$. This is not surprising because the cooling function scales as $\rho_{g}^{2}$, and so thermal balance can be achieved for a range of densities if the effective DF heating scales in the same way as cooling.

Nature may in fact provide this required scaling $\left(d \propto n_{e, 0}\right)$. For example, the number of galaxies per cluster is correlated with the cluster mass as $N_{\text {gal }} \propto M^{0.8}$ (Lin et al. 2004), with apparently little change at higher redshift (Lin et al. 2006), and the central electron density scales roughly as the cluster mass: $n_{e .0} \propto M$ (though with large scatter; Vikhlinin et al. 2006b; Zakamska \& Narayan 2003). Since we have incorporated the variation of all free parameters in Equation 19 into the single parameter $d$, it follows that $d \propto N_{\text {gal }}$. These additional scalings may thus in fact produce $d \propto n_{e, 0}^{\beta}$ with $\beta \sim 1$.

In order to investigate this explicitly, we have plotted the approximate locations of several Abell clusters in the $d-n_{e, 0}$ 

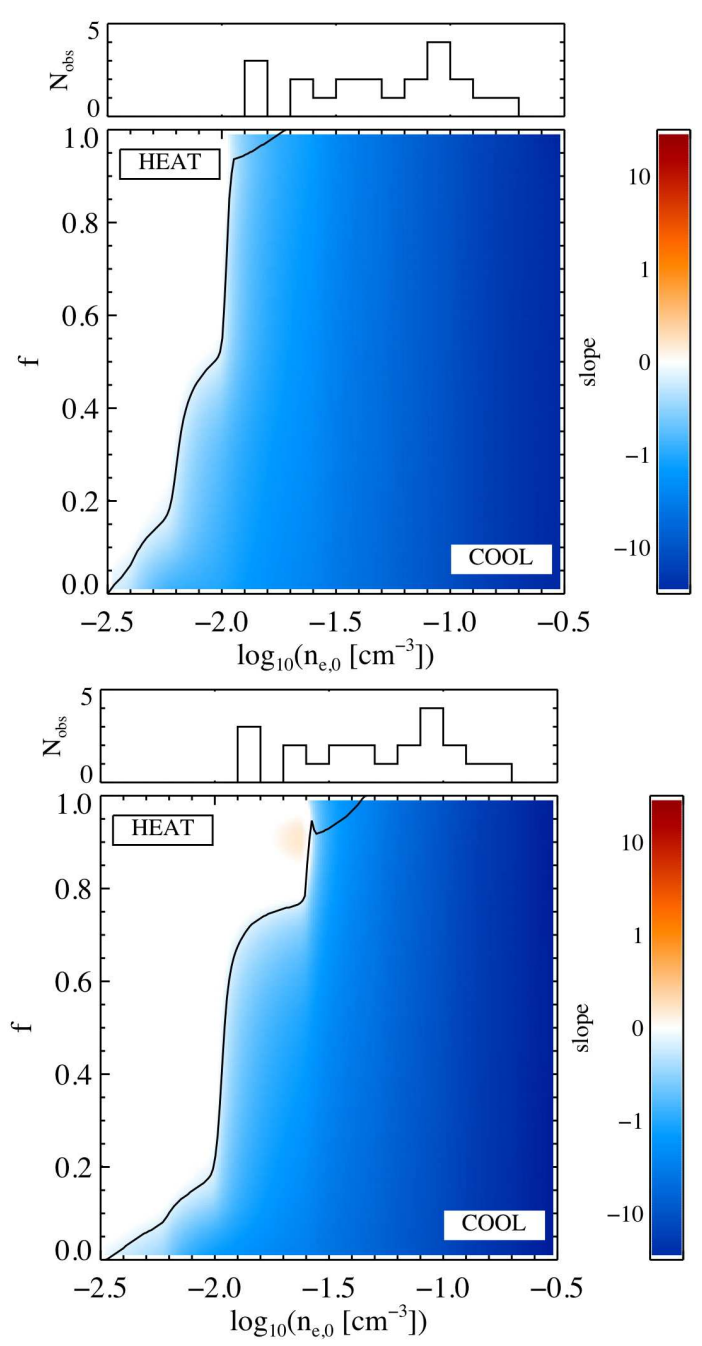

FIG. 6.- Same as Figure 4 except now only thermal conduction and radiative cooling are considered, and the clusters are initially all NCC. The normalization of the conductivity, $f$ (cf. Equation 14), is varied as a function of the initial central electron density $n_{e, 0}$. The initial gas temperatures are 5 $\mathrm{keV}$ (top panel) and $8 \mathrm{keV}$ (bottom panel).

plane in Figure 4, where the central electron density is estimated from Vikhlinin et al. (2006b) and the number of galaxies, $N_{\text {gal }}$, is provided by the luminosity functions of Lin et al. (2004). We have only included the dependence of $d$ on $N_{\mathrm{gal}}$ when estimating the different DF efficiencies for these clusters since this scaling is the dominant one. In other words, for these clusters we adopt our fiducial values relevant for DF heating except for $N_{\text {gal }}$, which we take from the literature. Moreover, the intracluster media of these clusters span a range in temperature (from $\sim 2-9 \mathrm{keV}$; Lin \& Mohr 2004). Since the region of thermal balance in Figure 4 is only a weak function of temperature, we include all the clusters on the same plot for simplicity. A trend of increasing $d$ with increasing $n_{e, 0}$ is evident, but the normalization is a factor of $\sim 3$ lower than the region where DF heating balances cooling. While a factor of three is probably within the uncertainty of our estimates of $d$ for these clusters, what is more important is the narrowness of the region. Our estimates of $d$ would not only have to be systematically off by a factor of $\sim 3$, but there would have to be rather extreme fine-tuning, especially at high $n_{e, 0}$, for DF heating to be generically be able to balance radiative losses. Moreover, even if clusters initially fell in this narrow range, DF heating would still respond to perturbations in $n_{e, 0}$ only linearly, while cooling would respond quadratically, and thus it is unlikely that clusters would remain in that narrow region for long.

The conclusion drawn from this section is straightforward and echoes the conclusion in Kim et al. (2005): DF heating, though an important reservoir of energy, cannot be the sole heating mechanism operating to offset radiative losses in the ICM. This is not because the energy available is insufficient but rather because there is only a narrow range of parameter space where DF heating can stably balance radiative cooling.

\subsection{Radiation \& Conduction}

Thermal conduction can in principle transport heat from the abundant thermal reservoir of the outer cluster gas to the inner cooling parts. Invoking conduction as a means to balance radiative losses in the ICM appears however to require a fair degree of fine tuning of the conductivity (Zakamska \& Naravan 2003; Kim \& Narayan 2003a; Pope et al. 2006; Guo \& Oh 2007). As demonstrated below, this problem is particularly acute in CC clusters where the observed temperature profile drops precipitously in the inner parts - too little conduction leads to a cooling catastrophe, while too much conduction tends to produce an isothermal core.

These issues are demonstrated graphically in Figure 5 where the evolution of the central temperature is plotted for both NCC (top panel) and CC (bottom panel) clusters, for a conductivity normalization $f=0.1$. Notice that the evolution is qualitatively different for NCC and CC clusters.

For NCC clusters, which are initially isothermal, only clusters with very low gas densities are marginally stable, while progressively more dense clusters quickly runaway. These trends can be understood with the aid of the conduction time:

$$
t_{\text {cond }} \equiv \frac{0.4}{f}\left(\frac{n_{e}}{0.01 \mathrm{~cm}^{-3}}\right)\left(\frac{\lambda}{100 \mathrm{kpc}}\right)^{2}\left(\frac{6 \mathrm{keV}}{T}\right)^{2.5} \mathrm{Gyr} \text {, }
$$

where $f$ is the conductivity normalization and $\lambda$ is the length scale over which temperature gradients are washed out within a conduction time (the inner region where the cooling time is shorter than a Hubble time is generally of order $100 \mathrm{kpc}$; Sanderson et al. 2006). It is clear that larger densities lead to longer conduction times ${ }^{2}$, and that for $f=0.1$, densities much greater than $n_{e, 0} \sim 10^{-2} \mathrm{~cm}^{-3}$ result in conduction times approaching the Hubble time, and much longer than the cooling time. For these high densities, conduction cannot forestall runaway cooling, as seen in Figure 5. Thermal conduction alone cannot therefore stably offset radiative losses in the intracluster media of NCC clusters.

The situation is somewhat more complex in CC clusters because there the initial temperature profile drops by a factor of $\sim 2-3$ within the inner $\sim 100 \mathrm{kpc}$. The bottom panel of Figure 5 shows the central temperature evolution for these clusters. For clusters with short conduction times, the inner (cooler) region isothermalizes to the temperature of the outer cluster region ( $\sim 6 \mathrm{keV}$ for these runs) before radiative effects become important. Once isothermal, the clusters then evolve more like the NCC clusters (top panel, Figure 5). CC clus-

2 The conduction time depends on density because, while conduction transports energy at fixed density, energy scales as $n_{e} T$, so that the conduction time scales as $n_{e}$. 
ters that are too dense have conduction times that are longer than the cooling times, and they thus cool catastrophically. These trends persist for the full range of conductivities explored herein $(0<f<1)$.

The trends evident for NCC clusters in the top panel of Figure 5 are shown for a wide range in conductivity normalizations and central electron densities in Figure 6. We have simulated NCC clusters with an initial temperature of $5 \mathrm{keV}$ (top panel) and $8 \mathrm{keV}$ (bottom panel) in order to demonstrate explicitly that our results are relatively insensitive to the ICM temperature. Unlike Figure 4 NCC clusters can only cool or remain in thermal balance, since conduction acting on an isothermal gas cannot produce a steadily increasing central temperature with time. It is clear that increasing the conductivity results in stable intracluster media over a wider range of initial central electron densities, but the range where cooling balances heating increases only mildly as the conduction normalization increases from $f \sim 0.2$ to $f \sim 1.0$.

The DF heating and conduction fine tuning problems are thus somewhat different. In the former case, too much DF heating will result in runaway heating, while in the latter, too much conduction simply isothermalizes the core. This is not acceptable because the majority of clusters show a factor of $\sim$ 2-3 temperature drop within their cores (e.g. Sanderson et al. 2006; Vikhlinin et al. 2006b). The implications are however the same: neither mechanism is generically able to reproduce observed temperature profiles.

It thus appears that previously proposed models for the ICM that construct clusters initially in thermal balance with conduction as the only heating source (Zakamska \& Narayan 2003; Kim \& Narayan 2003a; Guo \& Oh 2007) constitute a very narrow regime of parameter space and should thus not be considered realistic solutions to the cooling flow problem, unless a physical explanation for the fine-tuning is provided (see also the discussion in Guo \& Oh 2007).

\subsection{Radiation, DF Heating, \& Conduction}

In this section the thermal balance of the ICM is explored when both DF heating and conduction are operating as heating mechanisms.

Figure 7 plots the evolution of the central temperature for NCC and CC clusters with normalization of DF and conduction set to $d=1.0$ and $f=0.1$. Comparing this figure to Figures 5 and 3 highlights the stabilizing effects of the combination of DF heating and conduction. Note however that the evolved CC clusters are plagued by the same issues discussed in $\$ 3.3$ namely that while the combination of DF and conduction can maintain CC clusters in thermal balance, the evolved clusters do not preserve the factor of $\sim 2-3$ drop in the temperature profile characteristic of observed CC clusters. In other words, these $\mathrm{CC}$ clusters that do not catastrophically cool instead turn into NCC clusters, and thus these runs cannot explain the existence of observed CC clusters.

The increased thermal balance when combining DF heating with conduction is manifest in Figure 8, which shows the evolution of the central temperature of the ICM for initially NCC clusters with $T_{i, N C C}=6 \mathrm{keV}$ in the parameter space of initial central electron density and normalization of DF heating. This figure shows the effect of including conduction with normalization $f=0.1$ (top panel) and $f=0.5$ (bottom panel).

Upon comparing this figure to Figures 4 and 6 , it is clear that the combination of DF heating and conduction makes the ICM of NCC clusters considerably more thermally balanced than either mechanism acting alone. Indeed, for initial central

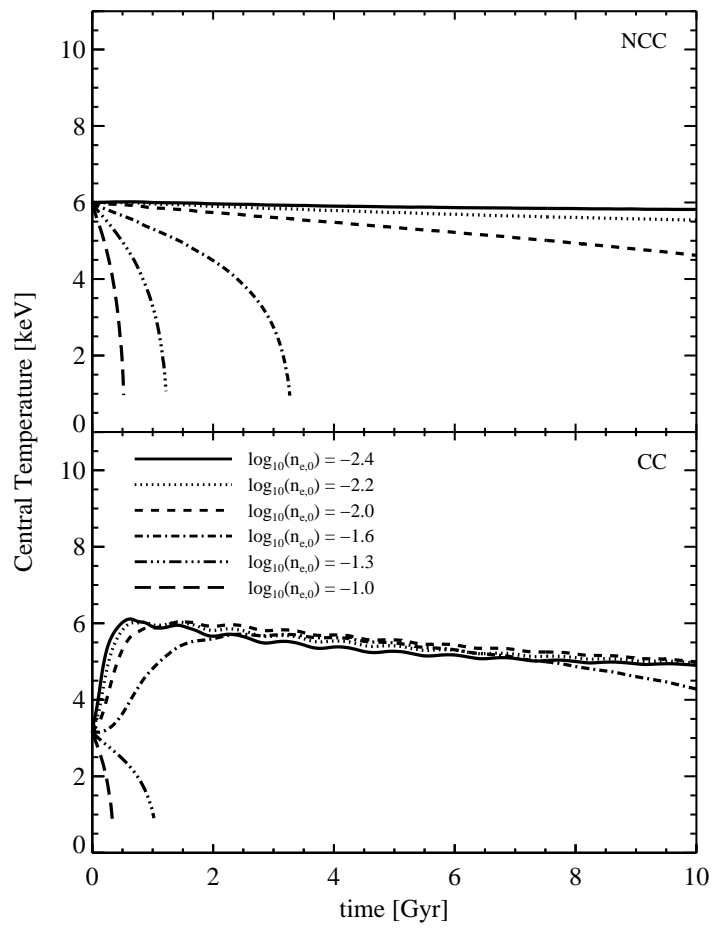

FIG. 7.- Evolution of the central temperature for simulations that include radiation, DF heating, and conduction. The DF heating and conductivity normalizations are $d=1.0$ and $f=0.1$, respectively. Top Panel: Initially NCC clusters. Bottom Panel: Initially CC clusters.

electron densities $n_{e, 0} \lesssim 0.02 \mathrm{~cm}^{-3}$ the ICM is thermally balanced for any plausible value of the DF heating normalization, and for reasonable, not fine-tuned, choices of conductivity.

However, it is still the case that for $n_{e, 0} \gtrsim 0.02 \mathrm{~cm}^{-3}$, no plausible amount of DF heating can offset the cooling catastrophe. Increasing the amount of conduction to $f=1.0 \mathrm{ex}-$ pands the zone of thermal balance to the right in Figure 8 only marginally. While some observed NCC clusters have a central density lying within the region of thermal balance in Figure 8, it is clear that for many observed clusters processes in addition to DF heating and conduction are at work in order to effectively balance radiative cooling.

These conclusions are rather insensitive to the initial temperature of the NCC and CC clusters. This might be expected because the cooling function scales as $T^{1 / 2}$ while DF heating scales at $T^{-1 / 2+p}$ with $p \sim 0$ (Kim et al. 2005). For example, decreasing the initial temperature in Figure 8 by a factor of two results in somewhat more thermally balanced clusters in the high density regime. However, the increased zone of thermal balance is narrow at high density and hence rather fine tuning would be required for an observed cluster to remain there. Moreover, observed clusters with larger central densities have higher rather than lower temperatures, and so the problem of generating equilibrium clusters is only exacerbated if the observed temperature-density relation is considered.

In sum, while it appears that the combination of DF and conduction can produce intracluster media in thermal balance for a modest range of central electron densities for NCC clusters, neither NCC clusters with moderately high central electron densities nor $\mathrm{CC}$ clusters of any density can be kept in 

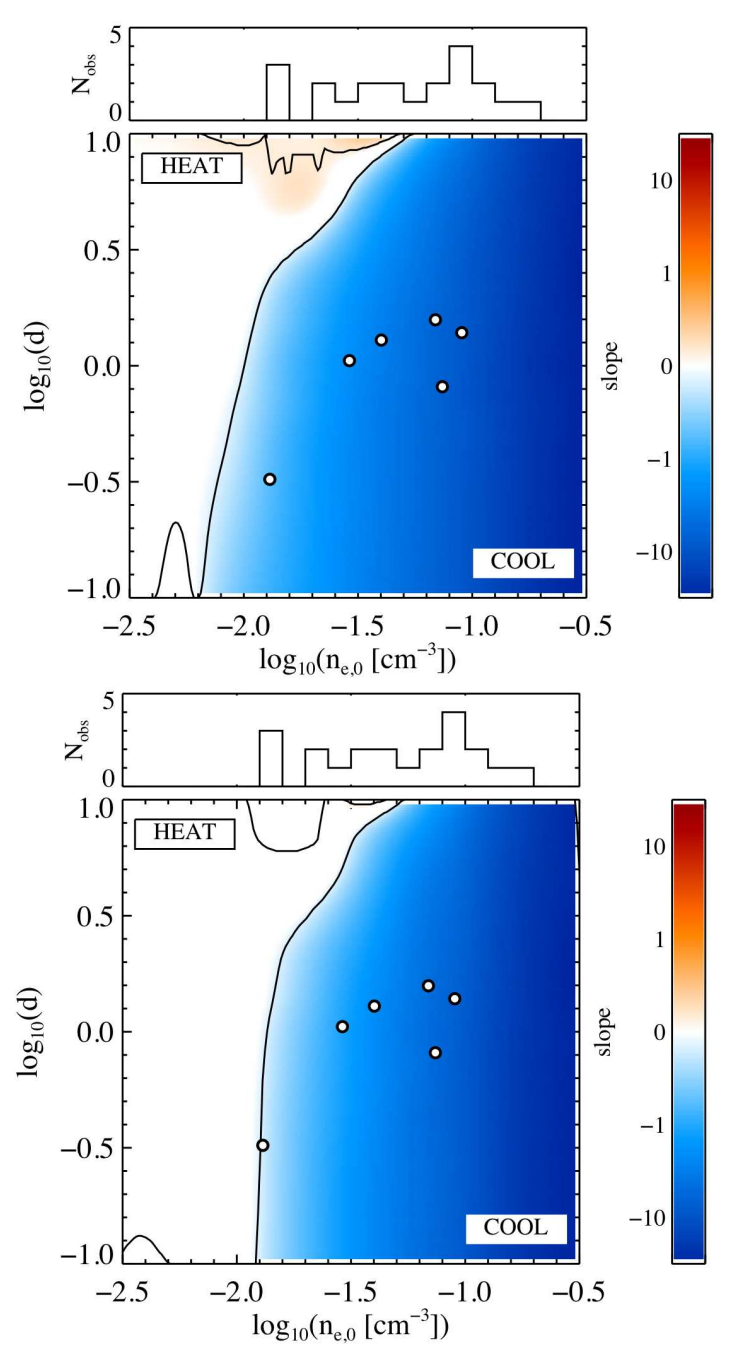

FIG. 8.- Same as Figure 4 now with DF heating, thermal conduction, and radiative cooling, for NCC clusters with $T_{i, N C C}=6 \mathrm{keV}$. Top Panel: NCC clusters with the conduction normalization set to $f=0.1$. Bottom Panel: NCC clusters with $f=0.5$. Note that even a small amount of conduction makes the clusters much more thermally stable than DF heating alone over a much wider range of central gas densities. As in Figure 4 the approximate locations of several Abell clusters are included (circles).

thermal balance for cosmological timescales with these mechanisms alone.

\subsection{The Impact of a Relativistic Fluid}

A relativistic fluid may be present in observed clusters, perhaps in the form of cosmic rays (Pfrommer \& Enßlin 2004; Sanders et al. 2005; Dunn \& Fabian 2006; Sanders \& Fabian 2007; Werner et al. 2007; Nakar et al. 2007). We show in the appendix that the addition of a small amount of relativistic pressure increases the thermal stability of a gas already in thermal balance (i.e. the growth of thermal perturbations is damped for intracluster media where the net heating of a parcel of gas is zero). In this section we explore the impact of a relativistic fluid on the thermal balance of the ICM.

As discussed in 2.3 , a relativistic fluid is included in our simulations by requiring that its pressure be a fixed fraction of the thermal pressure initially. The relativistic fluid is assumed to be massless and perfectly dynamically coupled to the gas.
Its subsequent adiabatic evolution is then governed by energy conservation. Recall that in our treatment the relativistic fluid provides no additional thermal energy to the system. The purpose of this experiment is thus to ascertain the potential importance of a relativistic fluid on the hydrodynamics of the ICM, not the thermodynamics.

We have run a series of simulations that span the range of parameter space shown in Figure 4 i.e. for a range in initial central electron densities and DF heating normalizations. In addition to varying these two variables, we have also varied the initial fraction of relativistic pressure, $\alpha$, from $10 \%$ to $40 \%$ of the total pressure, which is a fraction consistent with observations (Pfrommer \& Enßlin 2004; Sanders et al. 2005; Dunn \& Fabian 2006; Sanders \& Fabian 2007; Werner et al. 2007; Nakar et al. 2007). We find that the addition of relativistic pressure has a completely negligible effect on the thermal balance of the gas for the entire range of parameter space we have explored.

At first glance this is somewhat surprising since a thermal stability analysis indicates that a small amount of relativistic pressure should increase markedly the stability of a gas already in thermal balance (Cen 2005, see also $\widehat{A}$ ). The key difference is that stability analyses assume the gas to be in thermal balance, while the simulations we have run are almost never set initially in thermal balance. In the former case the classic Field (1965) instability (described in the Introduction) is suppressed because slightly over-dense fluid elements, which thus cool rapidly and hence lose thermal pressure support, contract less than they would otherwise thanks to the additional pressure support provided by the relativistic fluid. However, in the latter case, where the gas in not in thermal balance, the additional pressure support provided by the relativistic fluid is immaterial because the thermal runaway is caused by a more serious imbalance between heating and cooling, which the relativistic fluid, providing only pressure support and not energy, cannot remedy.

\section{DISCUSSION}

The results of the preceding section imply that both thermal conduction and DF heating are potentially significant heating processes in the ICM. In fact, for large regions of parameter space, these processes provide more than enough energy to offset radiative losses. However, we have found that they can neither produce nor maintain intracluster media in thermal balance over cosmological time-scales. In particular, these processes tend to generate either runaway cooling or runaway heating, in both cases producing temperature and density profiles of the ICM nowhere near those observed in nature (Sanderson et al. 2006; Vikhlinin et al. 2006b). These conclusions expand upon those of Brighenti \& Mathews (2002) who found that none of a large range of possible steady-state heating mechanisms could generically reproduce the observed properties of the ICM. For the case of DF heating, these results are driven largely by the fact that radiation losses scale as the gas density squared, while DF heating scales linearly with the density. Since observed intracluster media exhibit a wide range of densities, fine-tuning is required to prevent thermal runaways when DF is the dominant heating mechanism.

These conclusions have several implications, two of which we now discuss in detail. First, in light of our results from one dimensional simulations, we discuss the continued necessity of high-resolution, fully three-dimensional, cosmologically embedded hydrodynamic simulations for understanding the thermodynamics of the ICM. Second, we discuss a heat- 
ing mechanism that may plausibly generate and maintain the properties of observed intracluster media, since none of the mechanisms explored herein appear capable of doing so.

\subsection{The Cosmological Context}

Our approach has been to simulate in one dimension the evolution of the ICM when subjected to various heating mechanisms and radiative cooling in static, isolated clusters, where only the ICM was evolved. In the real Universe, however, clusters do not appear to evolve in isolation. Indeed, cosmological cold dark matter $N$-body simulations demonstrate that cluster-sized dark matter halos $\left(M \gtrsim 10^{14} M_{\odot}\right)$ continually accrete additional halos with a range of masses, many of which likely host galaxies. The mass growth rate is time-dependent, slowing at late times (e.g. Wechsler et al. 2002). The cosmological context within which clusters evolve may in fact play a significant role in their thermal history, as suggested by recent hydrodynamic simulations (e.g. Motl et al. 2004; Burns et al. 2007; Nagai et al. 2007; McCarthy et al. 2007b).

There are a variety of ways in which the cosmological setting can provide additional heating mechanisms. A generic class of such mechanisms may be called "gravitational" in the sense that the energy available for heating ultimately comes from the gravitational potential energy of infalling material. DF heating is one example of this class, but there are others. In particular, if small gaseous clumps are accreted, they may transfer their gravitational energy to the ICM via ram pressure drag and local shocks (e.g. Murray \& Lin 2004; McCarthy et al. 2007b; Khochfar \& Ostriker 2007; Dekel \& Birnboim 2007). Major mergers can significantly mix the ICM, effectively transporting the abundant reservoir of energy in the cluster outskirts to the cooler inner regions (e.g. Burns et al. 2007).

Recent observations have suggested that the fraction of $\mathrm{CC}$ clusters is increasing with time since at least $z \sim 1$ (Vikhlinin et al. 2006a; O'Hara et al. 2007). If gravitational heating plays a significant role in the thermal history of the ICM, then one may expect that as the accretion rate slows at late times (Wechsler et al. 2002), radiative losses would become increasingly dominant, in at least qualitative agreement with these recent observations. More generally, it is clear that the time-evolution of the abundance of observed CC clusters will provide strong, unique constraints on the importance of various heating mechanisms of the ICM.

While it may be appealing to invoke major mergers or other strong mixing processes as a means to transfer energy into the inner cooling regions of clusters, such processes have potential draw-backs. The most serious is that observed CC clusters have strong metallicity gradients within the cooling region (De Grandi \& Molendi 2001). If the metals are produced by type Ia $\mathrm{SNe}$ within the central galaxy then the timescale for generating the observed gradient is $\sim 5$ Gyr (Böhringer et al. 2004), which is much longer than the cooling time of the ICM in most observed clusters (Sanderson et al. 2006). Sedimentation may also contribute to the metallicity gradient on relevant timescales, but its relevance is much more uncertain because it can be highly suppressed in the presence of even modest magnetic fields (e.g. Fabian \& Pringle 1977). If mergers are capable of mixing the ICM such that the inner cooling region is effectively heated, it seems plausible that they may also destroy the metallicity gradient. Detailed numerical simulations are required to address these issues.

It is clear that $3 \mathrm{D}$ cosmologically embedded simulations are preferable to $1 \mathrm{D}$ simulations of the type presented herein because there are potentially relevant physical processes that cannot be adequately captured in the latter approach. While we have highlighted the potential importance of the cosmological context, it is also true that certain physical processes cannot be captured simply because our simulations are in 1D. For example, processes such as convection and the RayleighTaylor instability cannot be captured in a $1 D$ simulation. Additional insight into the cooling flow problem can thus be gained by simply extending the types of simulations we have run into $3 \mathrm{D}$.

In spite of these fact, the motivations for focusing on 1D simulations are several-fold. First, cosmologically embedded simulations are very time-consuming - a parameter space study similar to what we have presented in this work is not currently possible with such simulations. Second, DF is a resonant process (Tremaine \& Weinberg 1984), and so the particle requirement to adequately resolve this phenomenon may be considerably higher than what is achievable in the current generation of cosmologically embedded hydrodynamic simulations. It is not sufficient to resolve well the dark matter halo and subhalos. Since much of the DF heating is caused by the inspiralling of the much smaller stellar systems, a resolution small compared to the $\sim 10 \mathrm{kpc}$ half-light radii of giant ellipticals is required. Thus, while our approach to DF heating has been idealized, it was necessary to explore its possible effects in this parameterized manner because it is not clear that current 3D simulations are adequately resolving this phenomenon (see discussion in Faltenbacher et al. 2005; Naab et al. 2007). Finally, even with sufficient resolution, there are a number of physical effects that we have demonstrated are potentially relevant for the thermodynamics of the ICM that are not included in the majority of cosmologically embedded hydrodynamic simulations. Such phenomena include thermal conduction, which ultimately requires the inclusion of magnetic fields, type Ia supernovae energy injection, which is included in some but not all current simulations, and energy injection from accretion onto a central black-hole, which we now discuss in detail.

\subsection{A Self-Regulating Heating Mechanism}

In the present work we have focused on heating processes that are not generally thought of as "feedback" mechanisms. While DF heating is in principal a feedback mechanism since the heating becomes inefficient at very and low high Mach numbers, in practice this feedback is too weak to prevent runaway heating or cooling. The preceding discussion of the importance of the cosmological context suggests that additional gravitational energy is available that can contribute to the heating of the ICM. However, the incredibly short cooling times of many $\mathrm{CC}$ clusters $(\ll 1 \mathrm{Gyr})$ indicates that heating from without, via gravitational processes, must still be fine-tuned in order to prevent runaway cooling. Heating from within, via the release of energy from accretion onto a black hole, is in many ways more appealing because such a process is explicitly self-regulating.

Black hole accretion-mediated feedback mechanisms (e.g Ciotti \& Ostriker 2001; Ruszkowski \& Begelman 2002; Kaiser \& Binney 2003; Guo \& Oh 2007; Ciotti \& Ostriker 2007) are qualitatively different from the mechanisms we have explored. These self-regulating feedback processes are thought to work schematically as follows. As the gas begins to cool in the inner regions, its thermal pressure no longer provides sufficient support to the overlying material, and thus matter flows inward and eventually accretes onto the central 
black hole. The mechanical energy provided by AGN activity, which is proportional to the rate of mass infall, then heats up the surrounding gas until the thermal pressure, which is now increasing due to the energy injection, halts the inward flow of matter, thereby diminishing the accretion-driven energy injection. The cycle then begins again in a regulatory fashion, and the ICM thus neither heats nor cools catastrophically. It is this regulatory feedback processes which seems most promising in explaining the properties of observed intracluster media because the relevant physical parameters need not be tuned to any particular values for heating to balance cooling generically (see e.g. Guo \& Oh 2007).

A serious constraint on AGN-related feedback is that it may destroy the observed metallicity gradient in CC clusters if the energy deposition at the cluster center is sufficient to drive convection. Recently, Voit \& Donahue (2005) have shown that AGN feedback can be effective at balancing radiative cooling and maintaining the observed metallicity gradient if the AGN outbursts are rather gentle, occur every $\sim 10^{8}$ years and last in duration for $\sim 10^{7}$ years. It remains to be seen if this proposal can be confirmed with both numerical simulations and, ultimately, observations.

While AGN heating may be a promising candidate at generating and maintaining intracluster media in thermal balance over cosmological time-scales, simulations of the ICM which include it do so only in a rather simplistic fashion, in part because a detailed understanding of the physical processes involved in black hole accretion currently eludes us. The way in which energy is transfered from the black hole to the surrounding ICM is also obscure. Until these and related issues are better understood, we should continue to seek out other potential self-regulating heating processes that could potentially be relevant for the thermodynamics of the ICM. Nonetheless, varied observations of sound waves, bubbles, and strong radio emission (Bîrzan et al. 2004; Best et al. 2005; Fabian et al. 2006), predominantly in CC clusters, in addition to their very short cooling times (Sanderson et al. 2006), strongly suggests that AGN-related activity plays an important role in the thermodynamics of the ICM.

\section{SUMMARY}

We have presented the results from a series of 1D simulations aimed at understanding the importance of type Ia supernovae heating, thermal conduction and DF heating (due to the orbital motions of satellite galaxies) on the thermal properties of the intracluster media of clusters. Both initially noncooling core (NCC; i.e. isothermal) and cooling core (CC) clusters were simulated, for a wide range of initial central electron densities and gas temperatures.

Marginalizing over the uncertain efficiencies of DF heating and thermal conduction, it is clear that only NCC clusters with central electron densities $n_{e, 0} \lesssim 0.02 \mathrm{~cm}^{-3}$ can be maintained in thermal balance over a Hubble time if both DF and conduction operate; neither mechanism alone can generate generically stable ICM at these densities. At higher densities no reasonable amount of conduction or DF heating can prevent runaway cooling in NCC clusters.

The failure of the combination of conduction and DF heating at either generating or maintaining observed intracluster media is more pronounced for CC clusters, which have observed temperature profiles that decline by a factor of $\sim 2-3$ from the outer to inner regions. This temperature drop is extremely difficult to maintain in the face of thermal conduction in our simulations, because thermal conduction acts to erase temperature gradients. In fact, of the $\mathcal{O}\left(10^{3}\right)$ simulations run with a wide range of DF heating and conduction efficiencies and initial gas densities, none generated stable $\mathrm{CC}$ cluster profiles. Since CC clusters constitute $\sim 70 \%$ of observed clusters (e.g. Peres et al. 1998), we regard this failure as strong evidence that other heating processes in the ICM must be at work besides conduction and DF heating.

Our results demonstrate that there are numerous energy reservoirs capable of supplying enough energy to offset radiative losses. The crux of the cooling flow problem therefore lies not in finding one or more mechanisms capable of providing enough energy to the ICM, but rather in finding one or more mechanisms that can supply the energy in a way that maintains thermal equilibrium in the ICM over cosmological time-scales. Only low-density NCC clusters can be maintained with DF heating and conduction. The processes explored herein are not manifestly self-regulating, and they thus require fine-tuning in order to generate the observed properties of CC and high-density NCC intracluster media generically. For these types of observed intracluster media, it seems likely that an explicitly self-regulatory feedback process such as black hole accretion-powered energy injection (i.e. AGN feedback) is required.

CC gratefully acknowledges Jim Stone, Anatoly Spitkovsky, and Ian Parrish for extensive help and support with extensive numerical issues, and Andrey Kravtsov for many fruitful discussions. We thank Paul Bode, WoongTae Kim, Andrey Kravtsov, and Jim Stone for helpful comments on an earlier draft.

\section{REFERENCES}

Balbus, S. A. 1986, ApJ, 303, L79

Bell, E. F., McIntosh, D. H., Katz, N., \& Weinberg, M. D. 2003, ApJS, 149, 289

Best, P. N., Kauffmann, G., Heckman, T. M., Brinchmann, J., Charlot, S., Ivezić, Ž., \& White, S. D. M. 2005, MNRAS, 362, 25

Binney, J., Bibi, F. A., \& Omma, H. 2007, MNRAS, 377, 142

Binney, J. \& Cowie, L. L. 1981, ApJ, 247, 464

Binney, J. \& Tabor, G. 1995, MNRAS, 276, 663

Bîrzan, L., Rafferty, D. A., McNamara, B. R., Wise, M. W., \& Nulsen, P. E. J. 2004, ApJ, 607, 800

Blandford, R. D. \& Ostriker, J. P. 1978, ApJ, 221, L29

Bode, P., Ostriker, J. P., Weller, J., \& Shaw, L. 2007, ApJ, 663, 139

Böhringer, H., Matsushita, K., Churazov, E., Finoguenov, A., \& Ikebe, Y. 2004, A\&A, 416, L21

Borch, A., Meisenheimer, K., Bell, E. F., Rix, H.-W., Wolf, C., Dye, S., Kleinheinrich, M., Kovacs, Z., \& Wisotzki, L. 2006, A\&A, 453, 869
Boylan-Kolchin, M. \& Ma, C.-P. 2007, MNRAS, 374, 1227

Brighenti, F. \& Mathews, W. G. 2002, ApJ, 573, 542

-. 2003, ApJ, 587, 580

Brown, M. J. I., Dey, A., Jannuzi, B. T., Brand, K., Benson, A. J., Brodwin, M., Croton, D. J., \& Eisenhardt, P. R. 2007, ApJ, 654, 858

Brüggen, M. \& Kaiser, C. R. 2002, Nature, 418, 301

Bundy, K., Ellis, R. S., \& Conselice, C. J. 2005, ApJ, 625, 621

Burns, J. O., Hallman, E. J., Gantner, B., Motl, P. M., \& Norman, M. L. 2007, ArXiv:0708.1954, 708

Cen, R. 2005, ApJ, 620, 191

Chandran, B. D. G. \& Maron, J. L. 2004, ApJ, 602, 170

Cho, J. \& Lazarian, A. 2004, Journal of Korean Astronomical Society, 37, 557

Churazov, E., Brüggen, M., Kaiser, C. R., Böhringer, H., \& Forman, W. 2001, ApJ, 554, 261

Ciotti, L. \& Ostriker, J. P. 2001, ApJ, 551, 131 
-. 2007, ApJ, 665, 1038

Conroy, C., Wechsler, R. H., \& Kravtsov, A. V. 2006, ApJ, 647, 201

-. 2007, ApJ, 668, 826

Cowie, L. L. \& Binney, J. 1977, ApJ, 215, 723

De Grandi, S. \& Molendi, S. 2001, ApJ, 551, 153

Deiss, B. M. \& Just, A. 1996, A\&A, 305, 407

Dekel, A. \& Birnboim, Y. 2007, ArXiv:0707.1214, 707

Dennis, T. J. \& Chandran, B. D. G. 2005, ApJ, 622, 205

Dolag, K., Jubelgas, M., Springel, V., Borgani, S., \& Rasia, E. 2004, ApJ, 606, L97

Dunn, R. J. H. \& Fabian, A. C. 2006, MNRAS, 373, 959

Edge, A. C., Stewart, G. C., \& Fabian, A. C. 1992, MNRAS, 258, 177

El-Zant, A. A., Hoffman, Y., Primack, J., Combes, F., \& Shlosman, I. 2004a, ApJ, 607, L75

El-Zant, A. A., Kim, W.-T., \& Kamionkowski, M. 2004b, MNRAS, 354, 169

Enßlin, T. A. 2003, A\&A, 399, 409

Fabian, A. C. 1994, ARA\&A, 32, 277

-. 2003, MNRAS, 344, L27

Fabian, A. C. \& Nulsen, P. E. J. 1977, MNRAS, 180, 479

Fabian, A. C. \& Pringle, J. E. 1977, MNRAS, 181, 5P

Fabian, A. C., Sanders, J. S., Taylor, G. B., Allen, S. W., Crawford, C. S., Johnstone, R. M., \& Iwasawa, K. 2006, MNRAS, 366, 417

Fabian, A. C., Voigt, L. M., \& Morris, R. G. 2002, MNRAS, 335, L71

Faltenbacher, A., Kravtsov, A. V., Nagai, D., \& Gottlöber, S. 2005, MNRAS, 358, 139

Field, G. B. 1965, ApJ, 142, 531

Fontana, A. et al. 2006, A\&A, 459, 745

Fujita, Y. \& Suzuki, T. K. 2005, ApJ, 630, L1

Fukugita, M., Hogan, C. J., \& Peebles, P. J. E. 1998, ApJ, 503, 518

Gao, L., White, S. D. M., Jenkins, A., Stoehr, F., \& Springel, V. 2004, MNRAS, 355, 819

Girardi, M., Giuricin, G., Mardirossian, F., Mezzetti, M., \& Boschin, W. 1998, ApJ, 505, 74

Gonzalez, A. H., Zaritsky, D., \& Zabludoff, A. I. 2007, ApJ, 705

Greggio, L. 2005, A\&A, 441, 1055

Guo, F. \& Oh, S. P. 2007, ArXiv:0706.1274, 706

Hansen, S. M., Sheldon, E. S., Wechsler, R. H., \& Koester, B. P. 2007, ArXiv:0710.3780, 710

Just, A., Deiss, B. M., Kegel, W. H., Boehringer, H., \& Morfill, G. E. 1990, ApJ, 354, 400

Kaiser, C. R. \& Binney, J. 2003, MNRAS, 338, 837

Kelson, D. D., Zabludoff, A. I., Williams, K. A., Trager, S. C., Mulchaey, J. S., \& Bolte, M. 2002, ApJ, 576, 720

Khochfar, S. \& Ostriker, J. P. 2007, ArXiv e-prints: 0704.2418, 704

Kim, H. \& Kim, W.-T. 2007, ApJ, 665, 432

Kim, W.-T. 2007, ApJ, 667, L5

Kim, W.-T., El-Zant, A. A., \& Kamionkowski, M. 2005, ApJ, 632, 157

Kim, W.-T. \& Narayan, R. 2003a, ApJ, 596, 889

-. 2003b, ApJ, 596, L139

Kravtsov, A. V., Nagai, D., \& Vikhlinin, A. A. 2005, ApJ, 625, 588

Kravtsov, A. V. \& Yepes, G. 2000, MNRAS, 318, 227

Lima Neto, G. B., Gerbal, D., \& Márquez, I. 1999, MNRAS, 309, 481

Lin, Y.-T. \& Mohr, J. J. 2004, ApJ, 617, 879

Lin, Y.-T., Mohr, J. J., Gonzalez, A. H., \& Stanford, S. A. 2006, ApJ, 650, L99

Lin, Y.-T., Mohr, J. J., \& Stanford, S. A. 2003, ApJ, 591, 749

—. 2004, ApJ, 610, 745

Malyshkin, L. \& Kulsrud, R. 2001, ApJ, 549, 402

Mannucci, F., Della Valle, M., \& Panagia, N. 2006, MNRAS, 370, 773

Mannucci, F., Della Valle, M., Panagia, N., Cappellaro, E., Cresci, G., Maiolino, R., Petrosian, A., \& Turatto, M. 2005, A\&A, 433, 807

Markevitch, M., Vikhlinin, A., \& Forman, W. R. 2003, in Astronomical Society of the Pacific Conference Series, Vol. 301, Astronomical Society of the Pacific Conference Series, ed. S. Bowyer \& C.-Y. Hwang, 37

Masjedi, M. et al. 2006, ApJ, 644, 54

Mathews, W. G. \& Bregman, J. N. 1978, ApJ, 224, 308

Mathews, W. G., Faltenbacher, A., \& Brighenti, F. 2006, ApJ, 638, 659

McCarthy, I. G., Babul, A., Bower, R. G., \& Balogh, M. L. 2007a, ArXiv:0706.2768

McCarthy, I. G., Bower, R. G., Balogh, M. L., Voit, G. M., Pearce, F. R., Theuns, T., Babul, A., Lacey, C. G., \& Frenk, C. S. 2007b, MNRAS, 376, 497

Miller, L. 1986, MNRAS, 220, 713

Miniati, F., Ryu, D., Kang, H., \& Jones, T. W. 2001, ApJ, 559, 59
Motl, P. M., Burns, J. O., Loken, C., Norman, M. L., \& Bryan, G. 2004, ApJ, 606, 635

Murray, S. D. \& Lin, D. N. C. 2004, ApJ, 615, 586

Naab, T., Johansson, P. H., Ostriker, J. P., \& Efstathiou, G. 2007, ApJ, 658, 710

Nagai, D., Kravtsov, A. V., \& Vikhlinin, A. 2007, ApJ, 668, 1

Nakar, E., Milosavljevic, M., \& Nagai, D. 2007, ArXiv:0706.2881

Narayan, R. \& Medvedev, M. V. 2001, ApJ, 562, L129

Navarro, J. F., Frenk, C. S., \& White, S. D. M. 1997, ApJ, 490, 493

Nusser, A., Silk, J., \& Babul, A. 2006, MNRAS, 373, 739

O'Hara, T. B., Mohr, J. J., \& Sanderson, A. J. R. 2007, ArXiv:0710.5782, 710

Ostriker, E. C. 1999, ApJ, 513, 252

Ostriker, J. P. \& Hausman, M. A. 1977, ApJ, 217, L125

Parrish, I. J. \& Stone, J. M. 2007, Ap\&SS, 307, 77

Peres, C. B., Fabian, A. C., Edge, A. C., Allen, S. W., Johnstone, R. M., \& White, D. A. 1998, MNRAS, 298, 416

Peterson, J. R., Kahn, S. M., Paerels, F. B. S., Kaastra, J. S., Tamura, T., Bleeker, J. A. M., Ferrigno, C., \& Jernigan, J. G. 2003, ApJ, 590, 207

Peterson, J. R., Paerels, F. B. S., Kaastra, J. S., Arnaud, M., Reiprich, T. H. Fabian, A. C., Mushotzky, R. F., Jernigan, J. G., \& Sakelliou, I. 2001,

A\&A, 365, L104

Pfrommer, C. 2007, ArXiv:0707.1693

Pfrommer, C. \& Enßlin, T. A. 2004, A\&A, 413, 17

Pfrommer, C., Ensslin, T. A., \& Springel, V. 2007a, ArXiv:0707.1707

Pfrommer, C., Enßlin, T. A., Springel, V., Jubelgas, M., \& Dolag, K. 2007b, MNRAS, 378, 385

Pope, E. C. D., Pavlovski, G., Kaiser, C. R., \& Fangohr, H. 2006, MNRAS, 367, 1121

Press, W. H., Teukolsky, S. A., Vetterling, W. T., \& Flannery, B. P. 1992, Numerical recipes in FORTRAN. The art of scientific computing (Cambridge: University Press, |c1992, 2nd ed.)

Purcell, C. W., Bullock, J. S., \& Zentner, A. R. 2007, ApJ, 666, 20

Ruszkowski, M. \& Begelman, M. C. 2002, ApJ, 581, 223

Ruszkowski, M., Brüggen, M., \& Begelman, M. C. 2004a, ApJ, 611, 158 -. 2004b, ApJ, 615, 675

Ryu, D., Kang, H., Hallman, E., \& Jones, T. W. 2003, ApJ, 593, 599

Sánchez-Salcedo, F. J. \& Brandenburg, A. 2001, MNRAS, 322, 67

Sanders, J. S. \& Fabian, A. C. 2007, MNRAS, 951

Sanders, J. S., Fabian, A. C., \& Dunn, R. J. H. 2005, MNRAS, 360, 133

Sanderson, A. J. R., Ponman, T. J., Finoguenov, A., Lloyd-Davies, E. J., \& Markevitch, M. 2003, MNRAS, 340, 989

Sanderson, A. J. R., Ponman, T. J., \& O'Sullivan, E. 2006, MNRAS, 372, 1496

Sersic, J. L. 1968, Atlas de galaxias australes (Cordoba, Argentina: Observatorio Astronomico, 1968)

Spitzer, L. 1962, Physics of Fully Ionized Gases (Physics of Fully Ionized

Gases, New York: Interscience (2nd edition), 1962)

Sutherland, R. S. \& Dopita, M. A. 1993, ApJS, 88, 253

Tamura, T. et al. 2001, A\&A, 365, L87

Taylor, G. B., Fabian, A. C., \& Allen, S. W. 2002, MNRAS, 334, 769

Thomas, D., Maraston, C., Bender, R., \& Mendes de Oliveira, C. 2005, ApJ, 621,673

Tremaine, S. \& Weinberg, M. D. 1984, MNRAS, 209, 729

Tucker, W. H. \& Rosner, R. 1983, ApJ, 267, 547

van den Bosch, F. C., Weinmann, S. M., Yang, X., Mo, H. J., Li, C., \& Jing, Y. P. 2005, MNRAS, 361, 1203

Vikhlinin, A., Burenin, R., Forman, W. R., Jones, C., Hornstrup, A., Murray, S. S., \& Quintana, H. 2006a, astro-ph/0611438

Vikhlinin, A., Kravtsov, A., Forman, W., Jones, C., Markevitch, M., Murray, S. S., \& Van Speybroeck, L. 2006b, ApJ, 640, 691

Voigt, L. M. \& Fabian, A. C. 2004, MNRAS, 347, 1130

Voigt, L. M., Schmidt, R. W., Fabian, A. C., Allen, S. W., \& Johnstone, R. M. 2002, MNRAS, 335, L7

Voit, G. M. \& Donahue, M. 2005, ApJ, 634, 955

Wechsler, R. H., Bullock, J. S., Primack, J. R., Kravtsov, A. V., \& Dekel, A. 2002, ApJ, 568, 52

Werner, N., Kaastra, J. S., Takei, Y., Lieu, R., Vink, J., \& Tamura, T. 2007, A\&A, 468, 849

Xiang, F., Churazov, E., Dolag, K., Springel, V., \& Vikhlinin, A. 2007, MNRAS, 379, 1325

Zakamska, N. L. \& Narayan, R. 2003, ApJ, 582, 162 


\section{A. THERMAL STABILITY FOR GENERIC HEATING MECHANISMS IN THE PRESENCE OF A RELATIVISTIC FLUID}

The generalized Field criterion (Field 1965; Balbus 1986) states that a gas is thermally unstable to isobaric perturbations if:

$$
\left.\frac{\partial(\mathcal{L} / T)}{\partial T}\right|_{P}<0
$$

where $\mathcal{L}$ is the net loss function per unit mass defined such that $\rho_{g} \mathcal{L}=\Lambda-\Gamma$ where $\Lambda$ and $\Gamma$ are the cooling and heating rates per unit volume. In what follows the subscript " $g$ ", denoting gaseous quantities, will be omitted for brevity.

The following identity holds for a general gas in thermal equilibrium $(\rho \mathcal{L}=0)$ :

$$
\left.\frac{\partial(\mathcal{L} / T)}{\partial T}\right|_{P}=\frac{1}{\rho T}\left[\left.\frac{\partial(\rho \mathcal{L})}{\partial T}\right|_{\rho}+\left.\left.\frac{\partial \rho}{\partial T}\right|_{P} \frac{\partial(\rho \mathcal{L})}{\partial \rho}\right|_{T}\right]
$$

The first and third derivatives on the right-hand side are determined by the relevant heating and cooling mechanisms while the second is set by the various sources of pressure support. The following identity will be useful:

$$
\left.\frac{\partial \rho}{\partial T}\right|_{P}=-\left.\frac{\partial P}{\partial T}\right|_{\rho} /\left.\frac{\partial P}{\partial \rho}\right|_{T}
$$

Assume that the pressure support is provided by both the thermal pressure of the gas and a relativistic fluid:

$$
P=P_{\text {th }}+P_{\text {rel }}=K_{1} \rho T+P_{\text {rel }}(\rho),
$$

where $K_{1}$ is a constant and $P_{\text {rel }}$ is a function only of density. It then follows that

$$
\left.\frac{\partial P}{\partial T}\right|_{\rho}=K_{1} \rho
$$

and

$$
\left.\frac{\partial P}{\partial \rho}\right|_{T}=K_{1} T+\left.\frac{\partial P_{\mathrm{rel}}}{\partial \rho}\right|_{T}
$$

and hence:

$$
\left.\frac{\partial \rho}{\partial T}\right|_{P}=\frac{K_{1} \rho}{K_{1} T+\left.\frac{\partial P_{\mathrm{rel}}}{\partial \rho}\right|_{T}}=\frac{\rho}{T} \frac{P_{\mathrm{th}}}{P_{\mathrm{th}}+\left.\rho \frac{\partial P_{\mathrm{rel}}}{\partial \rho}\right|_{T}}=\frac{\rho}{T} \frac{\alpha^{\prime}}{\alpha^{\prime}+\left.\frac{\partial \ln P_{\mathrm{rel}}}{\partial \ln \rho}\right|_{T}},
$$

where in the last equality we have defined $\alpha^{\prime} \equiv P_{\text {th }} / P_{\text {rel }}$.

Now assume that cooling is dominated by thermal Bremsstrahlung radiation and that the heating has a simply power-law dependence on $\rho$ and $T$. Then:

$$
\rho \mathcal{L}=\Lambda_{0} \rho^{2} T^{1 / 2}-\Gamma_{0} \rho^{\beta} T^{-1 / 2+\delta},
$$

where $\Lambda_{0}$ and $\Gamma_{0}$ are constants and $\beta$ and $\delta$ are the power-law indices for heating, defined in a way that will prove useful below. After some algebra, and using the fact that $\rho \mathcal{L}=0$, we have

$$
\left.\frac{\partial(\rho \mathcal{L})}{\partial T}\right|_{\rho}=(1-\delta) \Lambda_{0} \rho^{2} T^{-1 / 2}
$$

and

$$
\left.\frac{\partial(\rho \mathcal{L})}{\partial \rho}\right|_{T}=(2-\beta) \Lambda_{0} \rho T^{1 / 2}
$$

Combining these with Equations $\mathrm{A} 2$ and $\mathrm{A} 7$ we have, after more algebra:

$$
\left.\frac{\partial(\mathcal{L} / T)}{\partial T}\right|_{P}=\Lambda_{0} \rho T^{-3 / 2}\left[(1-\delta)-(2-\beta) \frac{\alpha^{\prime}}{\alpha^{\prime}+\left.\frac{\partial \ln P_{\mathrm{rel}}}{\partial \ln \rho}\right|_{T}}\right] .
$$

The gas is thus thermally unstable if:

$$
\delta>\frac{(\beta-1) \alpha^{\prime}+\left.\frac{\partial \ln P_{\mathrm{rel}}}{\partial \ln \rho}\right|_{T}}{\alpha^{\prime}+\left.\frac{\partial \ln P_{\mathrm{rel}}}{\partial \ln \rho}\right|_{T}}=\frac{(\beta-1) \alpha^{\prime}+\gamma}{\alpha^{\prime}+\gamma}=\frac{(\beta-1)+\frac{\alpha}{1-\alpha} \gamma}{1+\frac{\alpha}{1-\alpha} \gamma},
$$

where we have assumed that $P_{\text {rel }} \propto \rho^{\gamma}$ and defined $\alpha \equiv P_{\text {rel }} / P_{\text {tot }}$.

This equation recovers the result found in Kim et al. (2005). There they explored the stability of DF heating, where $\beta=1$, in the absence of relativistic pressure $(\alpha=0)$. The instability criterion in this case is $\delta>0$. As shown in Kim et al. (2005), DF heating generally yields a temperature dependence such that $\delta>0$, and thus DF heating alone is thermally unstable. 
This equation also implies that heating sources which scale as $\rho^{2}$ are thermally stable if the temperature dependence scales as a power less than $1 / 2$, which is physically intuitive since $\rho^{2} T^{1 / 2}$ is the scaling of the cooling function. Moreover, if the heating source scales as $\rho^{2}$ then the relativistic fluid has no influence on the thermal stability. 